\title{
Hypersingular formulation for boundary stress evaluation revisited. Part 1: Smooth boundaries.
}

\author{
Alberto Salvadori \\ DICATA, University of Brescia, via Branze 43, 25123 Brescia, Italy
}

\begin{abstract}
This work focuses on the (HBIE) hypersingular boundary integral equation, also called traction equation, and on its use to evaluate the stress tensor in linear elasticity. When the field point is moved to the boundary, by means of a limit process, free terms come into play. As a common belief, they are due to the strongly singular kernel: indeed it is proved that the hypersingular kernel does not cause any free term when tractions are evaluated on smooth boundaries with respect to the boundary surface normal (when the concept of normal makes sense). The stress tensor along the boundary involves surfaces with normal differing from the boundary normal, too. In this case, free terms are proved to be generated also by the hypersingular kernel, aside from the regularity of the boundary: their analysis is the main goal of the present work.
\end{abstract}

Keywords

Hypersingular boundary integral equations; stress evaluation; free terms; boundary element method

\section{Introduction}

Boundary integral equations (BIEs) of a linear elastic problem in the framework of small strains and displacements stem from Somigliana's identity [1], which is the boundary integral representation of displacements at a point $\mathbf{x}$ inside an open domain $\Omega \in \mathbf{R}^{d}, d=2,3$, made of a homogeneous isotropic material. The traction operator can be applied to Somigliana's identity, in view of the regularity of the operators involved, thus obtaining the boundary integral representation of tractions $\mathbf{p}$ at an interior point $\mathbf{x} \in \Omega$ on a surface of normal $\mathbf{n}(\mathbf{x})$ [2]. Such a representation formula (called "hypersingular identity" (HI) in [3]) involves Green's functions (collected in matrices $\mathbf{G}_{p u}$ and $\mathbf{G}_{p p}$ ) which describe components $\left(p_{i}\right)$ of the traction vector $\mathbf{p}$ on a surface of normal $\mathbf{n}(\mathbf{x})$ due to: i) a unit force concentrated in space (point $\mathbf{y}$ ) and acting on the unbounded elastic space $\Omega_{\infty}$ in direction $j$; ii) a unit relative displacement concentrated in space (at a point $\mathbf{y}$ ), 
crossing a surface with normal $\mathbf{l}(\mathbf{y})$ and acting on the unbounded elastic space $\Omega_{\infty}$ (in direction $j$ ). Denoting with $\Gamma$ the boundary of $\Omega$, considering the quasi-static external actions: tractions $\overline{\mathbf{p}}(\mathbf{x})$ on $\Gamma_{p} \subset \Gamma$, displacements $\overline{\mathbf{u}}(\mathbf{x})$ on $\Gamma_{u}=\Gamma \backslash \Gamma_{p}$, and assuming zero domain forces $\overline{\mathbf{f}}(\mathbf{x})=\mathbf{0}$ in $\Omega$, the HI reads:

$$
\begin{aligned}
& \chi_{\Omega}(\mathbf{x}) \mathbf{p}(\mathbf{x})+\int_{\Gamma_{p}} \mathbf{G}_{p p}(\mathbf{r} ; \mathbf{n}(\mathbf{x}) ; \mathbf{l}(\mathbf{y})) \mathbf{u}(\mathbf{y}) \mathrm{d} \Gamma_{\mathbf{y}}+ \\
& \quad+\int_{\Gamma_{u}} \mathbf{G}_{p p}(\mathbf{r} ; \mathbf{n}(\mathbf{x}) ; \mathbf{l}(\mathbf{y})) \overline{\mathbf{u}}(\mathbf{y}) \mathrm{d} \Gamma_{\mathbf{y}}=\int_{\Gamma_{u}} \mathbf{G}_{p u}(\mathbf{r} ; \mathbf{n}(\mathbf{x})) \mathbf{p}(\mathbf{y}) \mathrm{d} \Gamma_{\mathbf{y}}+ \\
& \quad+\int_{\Gamma_{p}} \mathbf{G}_{p u}(\mathbf{r} ; \mathbf{n}(\mathbf{x})) \overline{\mathbf{p}}(\mathbf{y}) \mathrm{d} \Gamma_{\mathbf{y}}, \quad \mathbf{x} \notin \Gamma
\end{aligned}
$$

having set $\mathbf{r} \stackrel{\text { def }}{=} \mathbf{x}-\mathbf{y}$ and $\boldsymbol{\chi}_{\Omega}(\mathbf{x}) \stackrel{\text { def }}{=}\{\mathbf{I}$ if $\mathbf{x} \in \Omega, \mathbf{0}$ otherwise $\}$.

The traction BIE can be derived from (1) by performing the limit to the boundary $\Omega \ni \mathbf{x} \rightarrow \mathbf{x}_{0} \in \Gamma$. In the limit process, singularities of Green's functions are triggered off: kernel $\mathbf{G}_{p u}$ shows a strong singularity of $O\left(r^{-d+1}\right)$; kernel $\mathbf{G}_{p p}$ is usually called hypersingular, since it shows a singularity of $O\left(r^{-d}\right)$ greater than the dimension of the integral [4]. Moreover, the strongly singular kernel $\mathbf{G}_{p u}$ generates a free term $\mathbf{D}(\mathbf{x})$, that holds $1 / 2 \mathbf{I}$ for smooth boundaries. The traction equation, formulated on "smooth" boundaries, making use of the notions of Hadamard's finite part (HFP), see [5], and of Cauchy's principal value (CPV), see [6], reads as follows [7]:

$$
\begin{aligned}
\mathbf{D}(\mathbf{x}) \mathbf{p}(\mathbf{x}) & +f_{\Gamma_{p}} \mathbf{G}_{p p}(\mathbf{r} ; \mathbf{n}(\mathbf{x}) ; \mathbf{l}(\mathbf{y})) \mathbf{u}(\mathbf{y}) \mathrm{d} \Gamma_{\mathbf{y}}+ \\
& +f_{\Gamma_{u}} \mathbf{G}_{p p}(\mathbf{r} ; \mathbf{n}(\mathbf{x}) ; \mathbf{l}(\mathbf{y})) \overline{\mathbf{u}}(\mathbf{y}) \mathrm{d} \Gamma_{\mathbf{y}}=f_{\Gamma_{u}} \mathbf{G}_{p u}(\mathbf{r} ; \mathbf{n}(\mathbf{x})) \mathbf{p}(\mathbf{y}) \mathrm{d} \Gamma_{\mathbf{y}}+ \\
& +f_{\Gamma_{p}} \mathbf{G}_{p u}(\mathbf{r} ; \mathbf{n}(\mathbf{x})) \overline{\mathbf{p}}(\mathbf{y}) \mathrm{d} \Gamma_{\mathbf{y}}, \quad \mathbf{x} \in \Gamma
\end{aligned}
$$

In equation (2), $\mathbf{p}(\mathbf{x})$ indicates the traction at $\mathbf{x} \in \Gamma$ referring to the outward normal $\mathbf{l}(\mathbf{x})$, that is $\mathbf{p}(\mathbf{x})$ stands for $\mathbf{p}(\mathbf{x}, \mathbf{l}(\mathbf{x}))$. This statement implicitly requires smoothness of the boundary, because $\mathbf{l}(\mathbf{x})$ must be definite at $\mathbf{x} \in \Gamma^{1}$. In this sense the boundary needed to be smooth, so far: investigations on boundary smoothness and the traction equation, proofs of the nature of HFP, CPV, and free terms in the limit process approach, and a short literature review are provided in Sections 2 and 3.

The traction and displacement equations form a linear integral problem, whose solution (displacement $\mathbf{u}(\mathbf{y})$ and traction $\mathbf{p}(\mathbf{y})$ fields along the boundary) is usually approximated by the boundary element method [7]. The evaluation of the stress tensor is a fundamental "post-processing" task, often performed by means of the hypersingular BIE (HBIE) for displacement derivatives [9]. In many applications

\footnotetext{
${ }^{1}$ On the opposite, the boundary limit of the Somigliana's identity can be considered at a non smooth point $[8]$
} 


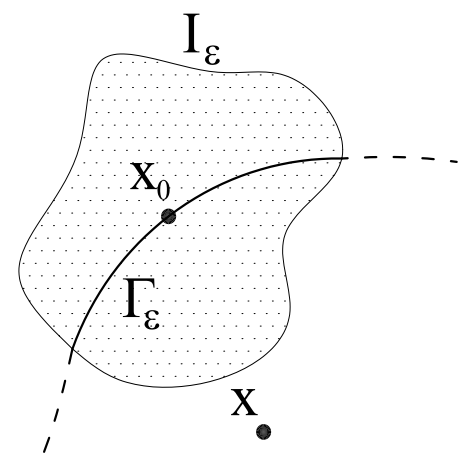

Figure 1: Boundary $\Gamma_{\varepsilon}$ and its complementary part.

(see e.g. [10], [11]) the most interesting points where to evaluate the stress tensor belong to the boundary and a natural way to get the stress tensor is by evaluating equation (1) with respect to a surface of normal $\mathbf{n}(\mathbf{x})$ orthogonal to the boundary normal $\mathbf{l}(\mathbf{x})$, thus obtaining the in-plane stress components by means of a limit to the boundary process $\Omega \ni \mathbf{x} \rightarrow \mathbf{x}_{0} \in \Gamma$.

The present work aims at analyzing the traction equation when direction $\mathbf{n}(\mathbf{x})$ differs from the boundary normal, what is missing so far, to the best of my knowledge, with the only partial exception of [12]. This note involves basic questions, such as: does the free term coefficient depend on the chosen direction $\mathbf{n}(\mathbf{x})$ ? does it depend on the smoothness of the boundary? does the hypersingular kernel contribute to it? are HFP and CPV well defined on any boundary? Such questions, some of which have also been tackled by other approaches ${ }^{2}$, are dealt with in section 2, for the free term coefficient due to the hypersingular kernel $\mathbf{G}_{p p}$, and in section 3 , with respect to the strongly singular kernel $\mathbf{G}_{p u}$.

In section 2 it is shown that $\mathbf{G}_{p p}$ always generates a free term coefficient, say $\mathbf{D}_{p p}(\mathbf{x}, \mathbf{n}(\mathbf{x}))$, that vanishes when the chosen direction $\mathbf{n}(\mathbf{x})$ approaches the outer normal $\mathbf{l}(\mathbf{x})$ on a smooth boundary. Similarly, the strongly singular kernel $\mathbf{G}_{p u}$ gives rise to a free term coefficient $\mathbf{D}_{p u}(\mathbf{x}, \mathbf{n}(\mathbf{x}))$ that yields $1 / 2 \mathbf{I}$ when the chosen direction $\mathbf{n}(\mathbf{x})$ approaches the outer normal $\mathbf{l}(\mathbf{x})$ on a smooth boundary. Furthermore, the hypersingular kernel generates free terms (called "additional" in [13]) on every boundary less smooth than $C^{2}$. Differently from $\mathbf{D}_{p p}(\mathbf{x}, \mathbf{n}(\mathbf{x}))$ such a free term, say $\mathbf{A}_{p p}(\mathbf{x}, \mathbf{n}(\mathbf{x}))$ as in [13], is merely due to the (lack of) smoothness of the boundary.

The usual way (see [8], [14], [9], [13], [15], [16]) to face up the free term analysis and the limit to the boundary process $\Omega \ni \mathbf{x} \rightarrow \mathbf{x}_{0} \in \Gamma$ excludes point $\mathbf{x}_{0} \in \Gamma$ by a neighborhood of arbitrary shapes, say $v_{\varepsilon}$, controlled by a parameter $\varepsilon ; v_{\varepsilon}$, the so-called "vanishing exclusion zone", vanishes as $\varepsilon \rightarrow 0$. The hypersingular identity (1) is considered on domain $\Omega \backslash v_{\varepsilon}$ (thus having $\chi_{\Omega \backslash v_{\varepsilon}}(\mathbf{x})=0$ ), integrals and

\footnotetext{
${ }^{2}$ as in [9], [13]: there it was proved in fact that hypersingular kernel do contributes to the free term coefficient for displacement derivatives (but it was not specified how) and implicitly that the free term coefficient does not depend on the chosen direction $\mathbf{n}(\mathbf{x})$
} 
their singularities are evaluated by suitably choosing $v_{\varepsilon}$ and finally the asymptotic analysis for $\varepsilon \rightarrow 0$ is performed. This approach stems from the identity (obtained from eq. (1))

$$
\begin{aligned}
\lim _{\varepsilon \rightarrow 0}\left\{\int_{\partial\left\{\Omega \backslash v_{\varepsilon}\right\}} \mathbf{G}_{p p}\left(\mathbf{x}_{0}-\mathbf{y} ; \mathbf{n}\left(\mathbf{x}_{0}\right) ; \mathbf{l}(\mathbf{y})\right) \mathbf{u}(\mathbf{y}) \mathrm{d} \Gamma_{\mathbf{y}}+\right. \\
\left.\quad-\int_{\partial\left\{\Omega \backslash v_{\varepsilon}\right\}} \mathbf{G}_{p u}\left(\mathbf{x}_{0}-\mathbf{y} ; \mathbf{n}\left(\mathbf{x}_{0}\right)\right) \overline{\mathbf{p}}(\mathbf{y}) \mathrm{d} \Gamma_{\mathbf{y}}\right\}=\mathbf{0}
\end{aligned}
$$

and is unsuitable for the goal of the present note: it does not permit to distinguish the contribution of each kernel to the free term and, ultimately, to analyze the dependence of the free term coefficient on direction $\mathbf{n}(\mathbf{x})$.

Accordingly, a different strategy is here pursued. Consider an open domain $\Omega \in \mathbf{R}^{2}$, and its boundary $\Gamma$, formed by a finite number of curve elements. $\Gamma$ is oriented by the field of the unit normal vectors $\mathbf{l}(\mathbf{y})$, uniquely defined for almost every $\mathbf{y} \in \Gamma$, with exceptions of corners. Take $\mathbf{x}_{0} \in \Gamma, I_{\varepsilon} \subset \mathbf{R}^{2}$ a neighborhood of it, and define $\Gamma_{\varepsilon} \stackrel{\text { def }}{=} \Gamma \bigcap I_{\varepsilon}$ (see figure 1). Equation (1) can be written by splitting $\Gamma$ in $\Gamma_{\varepsilon}$ and its complementary part. Free terms come out from integrals

$$
\begin{array}{cr}
\int_{\Gamma_{\varepsilon}} \mathbf{G}_{p p}(\mathbf{r} ; \mathbf{n}(\mathbf{x}) ; \mathbf{l}(\mathbf{y})) \mathbf{u}(\mathbf{y}) \mathrm{d} \Gamma_{\mathbf{y}} & \mathbf{x} \in \Omega \\
\int_{\Gamma_{\varepsilon}} \mathbf{G}_{p u}(\mathbf{r} ; \mathbf{n}(\mathbf{x})) \mathbf{p}(\mathbf{y}) \mathrm{d} \Gamma_{\mathbf{y}} & \mathbf{x} \in \Omega
\end{array}
$$

when $\Omega \ni \mathbf{x} \rightarrow \mathbf{x}_{0} \in \Gamma_{\varepsilon}$. The singular part of the previous integrals can be evaluated analytically, exploiting the regularity of the kernels and imposing suitable regularity to the solution.

With regard to the integral (3), suppose $\mathbf{u} \in C^{1, \alpha}\left(\Gamma_{\varepsilon}\right)$, that is $\mathbf{u}$ differentiable at $\mathbf{y} \in \Gamma_{\varepsilon}$ with its derivatives satisfying a Hölder condition. This requirement is not necessary, see [17], to the existence of the limit to the boundary of the hypersingular identity (1). However, $\mathbf{u} \in C^{1, \alpha}\left(\Gamma_{\varepsilon}\right)$ allows an asymptotic analysis of integral (3), by expanding $\mathbf{u}(\mathbf{y})$ around $\mathbf{x}_{0}$ as follows:

$$
\mathbf{u}(\mathbf{y})=\mathbf{u}\left(\mathbf{x}_{0}\right)+\left.\mathbf{J A C}_{\mathbf{y}}(\mathbf{u})\right|_{\mathbf{x}_{0}}\left(\mathbf{y}-\mathbf{x}_{0}\right)+O\left(\left\|\mathbf{y}-\mathbf{x}_{0}\right\|^{1+\alpha}\right)
$$

for any $0<\alpha \leq 1$. The Jacobian term is linked to the strain tensor $\boldsymbol{\epsilon}$ by the decomposition:

$$
\left.\mathbf{J A C}_{\mathbf{y}}(\mathbf{u})\right|_{\mathbf{x}_{0}}=\boldsymbol{\epsilon}\left(\mathbf{x}_{0}\right)+\operatorname{SKW}\left(\left.\mathbf{J A C}_{\mathbf{y}}(\mathbf{u})\right|_{\mathbf{x}_{0}}\right)
$$

By assuming that the skew-symmetric part (which describes only rigid body modes) vanishes, integral (3) becomes: 


$$
\begin{aligned}
\int_{\Gamma_{\varepsilon}} \mathbf{G}_{p p}(\mathbf{x}-\mathbf{y} ; \mathbf{n}(\mathbf{x}) ; \mathbf{l}(\mathbf{y})) \mathbf{u}(\mathbf{y}) \mathrm{d} \Gamma \\
=\int_{\Gamma_{\varepsilon}} \mathbf{G}_{p p}()\left[\mathbf{u}(\mathbf{y})-\mathbf{u}\left(\mathbf{x}_{0}\right)-\boldsymbol{\epsilon}\left(\mathbf{x}_{0}\right)\left(\mathbf{y}-\mathbf{x}_{0}\right)\right] \mathrm{d} \Gamma+ \\
\quad+\int_{\Gamma_{\varepsilon}} \mathbf{G}_{p p}() \mathrm{d} \Gamma \mathbf{u}\left(\mathbf{x}_{0}\right)+\int_{\Gamma_{\varepsilon}} \mathbf{G}_{p p}() \boldsymbol{\epsilon}\left(\mathbf{x}_{0}\right) \mathbf{y} \mathrm{d} \Gamma \quad \mathbf{x} \in \Omega
\end{aligned}
$$

In view of eq. (5) and of the hyper singularity of the kernel $\mathbf{G}_{p p}$, the function

$$
\mathbf{G}_{p p}(\mathbf{x}-\mathbf{y} ; \mathbf{n}(\mathbf{x}) ; \mathbf{l}(\mathbf{y}))\left[\mathbf{u}(\mathbf{y})-\mathbf{u}\left(\mathbf{x}_{0}\right)-\boldsymbol{\epsilon}\left(\mathbf{x}_{0}\right)\left(\mathbf{y}-\mathbf{x}_{0}\right)\right]
$$

is Lebesgue integrable on $\Gamma_{\epsilon}$ also when $\mathbf{x} \in \Omega$ moves to $\mathbf{x}_{0} \in \Gamma_{\varepsilon}$. Free terms arise therefore from the remaining terms, which will be dealt with in Section 2 by means of recently proposed formulae [18].

With regard to the integral (4), assume $\mathbf{p} \in C^{0, \alpha}\left(\Gamma_{\varepsilon}\right)$. This allows to expand $\mathbf{p}(\mathbf{y})$ around $\mathbf{x}_{0}$ as follows:

$$
\mathbf{p}(\mathbf{y})=\mathbf{p}\left(\mathbf{x}_{0}\right)+O\left(\left\|\mathbf{y}-\mathbf{x}_{0}\right\|^{\alpha}\right)
$$

for any $0<\alpha \leq 1$. In view of equation (7), integral (4) becomes:

$$
\begin{gathered}
\int_{\Gamma_{\varepsilon}} \mathbf{G}_{p u}(\mathbf{r} ; \mathbf{n}(\mathbf{x})) \mathbf{p}(\mathbf{y}) \mathrm{d} \Gamma=\int_{\Gamma_{\varepsilon}} \mathbf{G}_{p u}()\left[\mathbf{p}(\mathbf{y})-\mathbf{p}\left(\mathbf{x}_{0}\right)\right] \mathrm{d} \Gamma+ \\
+\int_{\Gamma_{\varepsilon}} \mathbf{G}_{p u}() \mathrm{d} \Gamma \mathbf{p}\left(\mathbf{x}_{0}\right) \quad \mathbf{x} \in \Omega
\end{gathered}
$$

In view of eq. (7) and of the strong singularity of the kernel $\mathbf{G}_{p u}$, the function

$$
\mathbf{G}_{p u}(\mathbf{x}-\mathbf{y} ; \mathbf{n}(\mathbf{x}))\left[\mathbf{p}(\mathbf{y})-\mathbf{p}\left(\mathbf{x}_{0}\right)\right]
$$

is integrable on $\Gamma_{\epsilon}$ also when $\mathbf{x} \in \Gamma_{\varepsilon}$. Free terms arise therefore from the remaining term, which will be considered in Section 3.

By this approach the contributions of the hypersingular kernel, $\mathbf{A}_{p p}(\mathbf{x}, \mathbf{n}(\mathbf{x}))$ and $\mathbf{D}_{p p}(\mathbf{x}, \mathbf{n}(\mathbf{x}))$, and of the strongly singular kernel $\mathbf{D}_{p u}(\mathbf{x}, \mathbf{n}(\mathbf{x}))$ to the free term coefficient $\mathbf{D}(\mathbf{x})$ are separately obtained; they depend on the elastic properties of domain $\Omega$ and on the selected direction $\mathbf{n}\left(\mathbf{x}_{0}\right)$ at point $\mathbf{x}_{0} \in \Gamma_{\varepsilon}$. The free term coefficient $\mathbf{D}(\mathbf{x})$ is a linear combination of $\mathbf{D}_{p p}(\mathbf{x}, \mathbf{n}(\mathbf{x}))$ and of $\mathbf{D}_{p u}(\mathbf{x}, \mathbf{n}(\mathbf{x}))$ :

$$
\mathbf{D}(\mathbf{x})=\mathbf{I}+\mathbf{D}_{p p}(\mathbf{x}, \mathbf{n}(\mathbf{x}))-\mathbf{D}_{p u}(\mathbf{x}, \mathbf{n}(\mathbf{x}))
$$

In Section 4 it is shown that, for smooth boundaries, $\mathbf{D}(\mathbf{x})$ is independent on the direction $\mathbf{n}(\mathbf{x})$ and on the material properties, and it holds again $1 / 2 \mathbf{I}$ for any direction. Accordingly, equation (2) holds as it stands for any direction $\mathbf{n}(\mathbf{x})$ on 
smooth boundaries, provided that $\mathbf{p}(\mathbf{x})$ refers to the direction $\mathbf{n}(\mathbf{x})$. Such a result is extended to non-smooth boundaries in a companion paper [19]. In that case, the free term coefficient is shown to depend on the material properties of the body, on the surface normal $\mathbf{n}(\mathbf{x})$ and on the angle at the corner point.

It is worth noting that the property of independence of $\mathbf{D}(\mathbf{x})$ on the direction $\mathbf{n}(\mathbf{x})$ and on the material properties of the domain holds for the exact solution fields but it is generally not satisfied by their approximation. A natural question comes into play, does the discretization play a role in the expression of the free term coefficient? Section 5 will show that the free term cannot be taken as $1 / 2 \mathbf{I}$ in the stress tensor evaluation, even for smooth boundaries. In fact, a convergent sequence of field points $\Omega \ni \mathbf{x}_{n} \rightarrow \mathbf{x}_{0} \in \Gamma$ is considered, together with the stress tensor $\boldsymbol{\sigma}\left(\mathbf{x}_{n}\right)$ : it is proved that $\boldsymbol{\sigma}\left(\mathbf{x}_{n}\right) \nrightarrow \boldsymbol{\sigma}\left(\mathbf{x}_{0}\right)$ when $\boldsymbol{\sigma}\left(\mathbf{x}_{0}\right)$ is evaluated by means of $\mathbf{D}(\mathbf{x})=1 / 2 \mathbf{I}$ on a smooth boundary. The actual value of the free term due to the discretization is provided.

\section{Free terms analysis for the hypersingular kernel}

This section aims at analyzing the limit $(\mathrm{x} \in \Omega)$

$$
\lim _{\mathbf{x} \rightarrow \mathbf{x}_{0}}\left\{\int_{\Gamma_{\varepsilon}} \mathbf{G}_{p p}(\mathbf{r} ; \mathbf{n}(\mathbf{x}), \mathbf{l}(\mathbf{y})) \mathrm{d} \Gamma_{\mathbf{y}} \mathbf{u}\left(\mathbf{x}_{0}\right)+\int_{\Gamma_{\varepsilon}} \mathbf{G}_{p p}(\mathbf{r} ; \mathbf{n}(\mathbf{x}), \mathbf{l}(\mathbf{y})) \boldsymbol{\epsilon}\left(\mathbf{x}_{0}\right) \mathbf{y} \mathrm{d} \Gamma_{\mathbf{y}}\right\}
$$

providing different smoothness to the curve $\Gamma_{\varepsilon}$, by changing the support of the integrals (9) from the curve $\Gamma_{\varepsilon}$ to the interval $I_{\varepsilon}$; thereafter, they will be evaluated by means of analytical integration formulae that have been presented in $[18]^{3}$. In changing the support, the order of the induced approximation on the integral

$$
\lim _{\mathbf{x} \rightarrow \mathbf{x}_{0}}\left\{\int_{\Gamma_{\varepsilon}} \mathbf{G}_{p p}(\mathbf{r} ; \mathbf{n}(\mathbf{x}), \mathbf{l}(\mathbf{y})) \mathrm{d} \Gamma_{\mathbf{y}} \quad \mathbf{x} \in \Omega\right\}
$$

depends on the smoothness of the boundary. In section 2.1 suitable smoothness conditions are considered to let the approximation be $O(\varepsilon)$. Starting from section 2.2 , smoothness conditions lead to an $O(1)$ approximation, thus a free term.

With regard to the limit

$$
\lim _{\mathbf{x} \rightarrow \mathbf{x}_{0}}\left\{\int_{\Gamma_{\varepsilon}} \mathbf{G}_{p p}(\mathbf{r} ; \mathbf{n}(\mathbf{x}), \mathbf{l}(\mathbf{y})) \boldsymbol{\epsilon}\left(\mathbf{x}_{0}\right) \mathbf{y} \mathrm{d} \Gamma_{\mathbf{y}} \quad \mathbf{x} \in \Omega\right\}
$$

the order of the induced approximation always turns out to be $O(\varepsilon)$ on smooth boundaries. Nevertheless, free terms may arise which have a different nature.

\footnotetext{
${ }^{3}$ For the formal complexity of such formulae, the computer code MATHEMATICA, release 4, has been extensively used.
} 


\subsection{Smooth boundaries}

Let $\tilde{\mathbf{y}}(s)=\left\{\tilde{y}_{1}(s), \tilde{y}_{2}(s)\right\}, 0 \leq s \leq l$ be the parametric equations of curve $\Gamma_{\varepsilon}$ with $s$ denoting the curvilinear abscissa; assume $\tilde{\mathbf{y}}(\cdot)$ differentiable in $0 \leq s \leq l$. Consider a local coordinate system $\mathcal{L}$ centered at $\mathbf{x}_{0} \in \Gamma_{\varepsilon}$, as in figure 2-a, with axis $y_{1}, y_{2}$ tangent and normal to $\Gamma_{\varepsilon}$ at $\mathbf{x}_{0}$, respectively. Point $\mathbf{x}_{0}$ is selected such that the parametric equations of $\Gamma_{\varepsilon}$ with respect to $y_{1} \operatorname{read} \mathbf{y}\left(y_{1}\right)=\left\{y_{1}, y_{2}\left(y_{1}\right)\right\}, y_{1} \in$ $I_{\varepsilon} \stackrel{\text { def }}{=}[-\varepsilon, \varepsilon]$. Define with $\mathbf{z} \stackrel{\text { def }}{=}\left\{y_{1}, 0\right\}$, as in figure 2-b, and with $B_{\tilde{\varepsilon}}$ a ball of radius $\tilde{\varepsilon}$ centered at $\mathbf{x}=\mathbf{0}$; assume $\mathbf{x} \in \Omega$ and the following:

Hypotheses 2.1 Let $\Gamma_{\varepsilon}$ be defined by $y_{2}\left(y_{1}\right)=y_{1}^{\alpha}, \alpha \geq 3$

For being $y_{2}^{\prime}(0)=0$ by definition, all results based on hypothesis 2.1 hold also for all $y_{2}(\cdot)$ that admit a Taylor expansion around $y_{1}=0$ with $y_{2}^{\prime \prime}(0)=0$. The following propositions hold.

Lemma 2.1 For any bounded function $f(\mathbf{x}, \mathbf{y})$ in $B_{\tilde{\varepsilon}} \times \Gamma_{\varepsilon}$ :

$$
\lim _{\mathbf{x} \rightarrow \mathbf{0}} \int_{-\varepsilon}^{\varepsilon} f(\mathbf{x}, \mathbf{y})\left(\frac{1}{\|\mathbf{x}-\mathbf{y}\|^{2}}-\frac{1}{\|\mathbf{x}-\mathbf{z}\|^{2}}\right) \mathrm{d} y_{1}=O(\varepsilon)
$$

Proof: The function $g\left(\mathbf{x}, y_{1}\right) \stackrel{\text { def }}{=} \frac{1}{\|\mathbf{x}-\mathbf{y}\|^{2}}-\frac{1}{\|\mathbf{x}-\mathbf{z}\|^{2}}$ is continuous in the set $B_{\tilde{\varepsilon}} \times I_{\varepsilon}$ if (and only if) $\alpha \geq 3$. It turns out therefore that [20]

$$
\lim _{\mathbf{x} \rightarrow \mathbf{0}} \int_{-\varepsilon}^{\varepsilon} g\left(\mathbf{x}, y_{1}\right) \mathrm{d} y_{1}=\int_{-\varepsilon}^{\varepsilon} \frac{1}{\|\mathbf{y}\|^{2}}-\frac{1}{\|\mathbf{z}\|^{2}} \mathrm{~d} y_{1}
$$

As a consequence, for any bounded function $f(\mathbf{x}, \mathbf{y})$ in $B_{\tilde{\varepsilon}} \times \Gamma_{\varepsilon}$ :

$$
\lim _{\mathbf{x} \rightarrow \mathbf{0}} \int_{-\varepsilon}^{\varepsilon} f(\mathbf{x}, \mathbf{y})\left(\frac{1}{\|\mathbf{x}-\mathbf{y}\|^{2}}-\frac{1}{\|\mathbf{x}-\mathbf{z}\|^{2}}\right) \mathrm{d} y_{1} \leq \sup _{B_{\tilde{\varepsilon}} \times \Gamma_{\varepsilon}}|f(\mathbf{x}, \mathbf{y})| \int_{-\varepsilon}^{\varepsilon}\left|\frac{1}{\|\mathbf{y}\|^{2}}-\frac{1}{\|\mathbf{z}\|^{2}}\right| \mathrm{d} y_{1}
$$

and the thesis follows by the mean value theorem.

Corollary 2.1.1 It holds:

$$
\lim _{\mathbf{x} \rightarrow \mathbf{0}} \int_{-\varepsilon}^{\varepsilon} \frac{f(\mathbf{x}, \mathbf{y})}{\|\mathbf{x}-\mathbf{y}\|^{2}} \mathrm{~d} y_{1}=\lim _{\mathbf{x} \rightarrow \mathbf{0}} \int_{-\varepsilon}^{\varepsilon} \frac{f(\mathbf{x}, \mathbf{y})}{\|\mathbf{x}-\mathbf{z}\|^{2}} \mathrm{~d} y_{1}+O(\varepsilon)
$$

Remarks to lemma 2.1

- It is worth noting that in equation (10) it holds:

$$
\frac{1}{\|\mathbf{y}\|^{2}}-\frac{1}{\|\mathbf{z}\|^{2}}=\frac{y_{1}^{2(\alpha-2)}}{1+y_{1}^{2(\alpha-1)}}
$$

which is integrable on $I_{\varepsilon}$ if (and only if) $\alpha>3 / 2$. The interesting point in equation (10) does not lie on the integrability of the function when $\alpha<3$, nor on the existence of the limit; instead, it lies on the convergence of the limit to the r.h.s. integral. 
- The function $\mathbf{G}_{p p}(\mathbf{x}-\mathbf{y} ; \mathbf{n}(\mathbf{x}) ; \mathbf{l}(\mathbf{y})) r^{2}$ is bounded around $\mathbf{x}=\mathbf{0}$ for all $\alpha$, whence the interest in the lemma 2.1 .
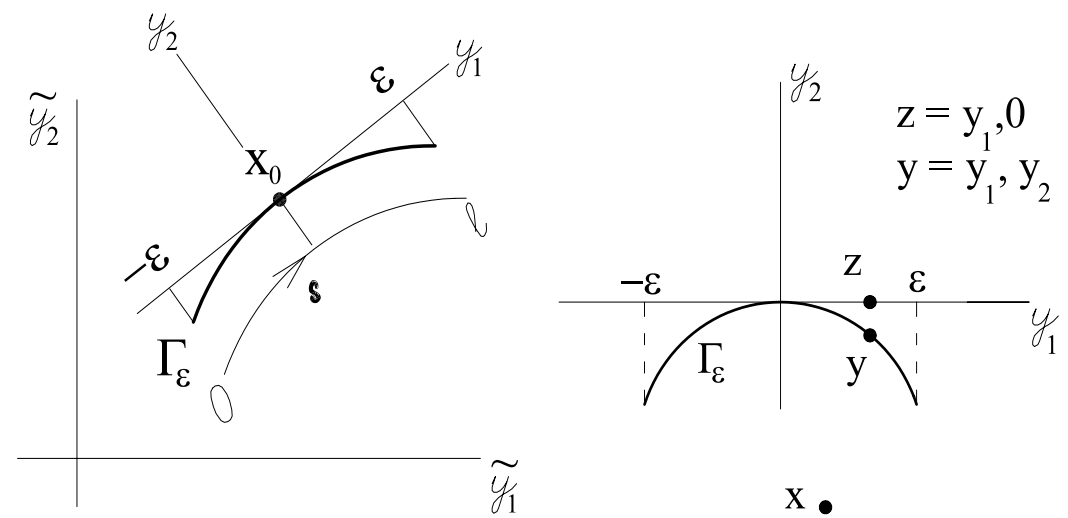

Figure 2: Global and local references on $\Gamma_{\varepsilon}$ for smooth boundaries.

Lemma 2.2 Denote by $f(\mathbf{x}, \mathbf{y})=\mathbf{G}_{p p}(\mathbf{x}-\mathbf{y} ; \mathbf{n}(\mathbf{x}) ; \mathbf{l}(\mathbf{y}))\|\mathbf{x}-\mathbf{y}\|^{2} \sqrt{1+y_{2}^{\prime 2}}$. It holds:

$$
\lim _{\mathbf{x} \rightarrow \mathbf{x}_{0}} \int_{-\varepsilon}^{\varepsilon} \frac{f(\mathbf{x}, \mathbf{y})-f(\mathbf{x}, \mathbf{z})}{\|\mathbf{x}-\mathbf{z}\|^{2}} \mathrm{~d} y_{1}=O(\varepsilon)
$$

Proof: $\quad$ By direct substitution, it comes out that $\frac{f(\mathbf{x}, \mathbf{y})-f(\mathbf{x}, \mathbf{z})}{\|\mathbf{x}-\mathbf{z}\|^{2}}$ is bounded on $B_{\tilde{\varepsilon}} \times \Gamma_{\varepsilon}$ (respectively: continuous) if $\alpha \geq 3$ (respectively: $\alpha>3$ ).

Corollary 2.2.1 It holds:

$$
\lim _{\mathbf{x} \rightarrow \mathbf{x}_{0}} \int_{-\varepsilon}^{\varepsilon} \frac{f(\mathbf{x}, \mathbf{y})}{\|\mathbf{x}-\mathbf{z}\|^{2}} \mathrm{~d} y_{1}=\lim _{\mathbf{x} \rightarrow \mathbf{x}_{0}} \int_{-\varepsilon}^{\varepsilon} \frac{f(\mathbf{x}, \mathbf{z})}{\|\mathbf{x}-\mathbf{z}\|^{2}} \mathrm{~d} y_{1}+O(\varepsilon)
$$

By taking $\mathbf{x} \notin \Gamma_{\varepsilon}$, lemmas 2.1 and 2.2 permit to "move" the integral from curve $\Gamma_{\varepsilon}$ to interval $I_{\varepsilon}$, controlling the order of the approximation:

$$
\begin{gathered}
\int_{\Gamma_{\varepsilon}} \mathbf{G}_{p p}(\mathbf{r} ; \mathbf{n}(\mathbf{x}), \mathbf{l}(\mathbf{y})) \mathrm{d} \Gamma_{\mathbf{y}}=\int_{-\varepsilon}^{\varepsilon} \mathbf{G}_{p p}(\mathbf{r} ; \mathbf{n}(\mathbf{x}), \mathbf{l}(\mathbf{y})) \sqrt{1+y_{2}^{\prime}\left(y_{1}\right)^{2}} \mathrm{~d} y_{1} \\
\stackrel{(\text { Lemma. 2.1) }}{=} \int_{-\varepsilon}^{\varepsilon} \frac{\mathbf{G}_{p p}(\mathbf{r} ; \mathbf{n}(\mathbf{x}), \mathbf{l}(\mathbf{y}))\|\mathbf{x}-\mathbf{y}\|^{2} \sqrt{1+y_{2}^{\prime}\left(y_{1}\right)^{2}}}{\|\mathbf{x}-\mathbf{z}\|^{2}} \mathrm{~d} y_{1}+O(\varepsilon) \\
\stackrel{(\text { Lemma. 2.2) }}{=} \int_{-\varepsilon}^{\varepsilon} \mathbf{G}_{p p}(\mathbf{x}-\mathbf{z} ; \mathbf{n}(\mathbf{x}), \mathbf{l}(\mathbf{z})) \mathrm{d} y_{1}+O(\varepsilon)
\end{gathered}
$$


It is quite natural that the approximation depends on exponent $\alpha$, because curve $\Gamma_{\varepsilon}$ approaches interval $I_{\varepsilon}$ when $\alpha$ becomes larger and larger. All introduced results lead to the following:

Proposition 2.1 For solving integral (9) on $C^{3}$ boundaries, provided that $y_{2}^{\prime \prime}(0)=$ 0 , it is sufficient to solve the following integrals:

$$
\int_{x_{1}-\varepsilon}^{x_{1}+\varepsilon} \mathbf{G}_{p p}(\mathbf{r} ; \mathbf{n}(\mathbf{x}) ; \mathbf{l}(\mathbf{y})) r_{1}^{i} \mathrm{~d} r_{1} \quad i=0,1 \quad \mathbf{x} \in \Omega
$$

Proof: Denoting with $\mathbf{e}_{1}$ the unit vector associated to the $y_{1}$-axis, in $\mathcal{L}$ one has $\boldsymbol{\epsilon}\left(\mathbf{x}_{0}\right) \mathbf{y}=y_{1} \boldsymbol{\epsilon}\left(\mathbf{x}_{0}\right) \mathbf{e}_{1}+O\left(y_{1}^{2}\right)$. To evaluate equation (9), one therefore deals with the following integrals:

$$
\int_{-\varepsilon}^{\varepsilon} \mathbf{G}_{p p}(\mathbf{x}-\mathbf{z} ; \mathbf{n}(\mathbf{x}) ; \mathbf{l}(\mathbf{z})) y_{1}^{i} \mathrm{~d} y_{1} \quad i=0,1 \quad \mathbf{x} \in \Omega
$$

that can be performed by means of the variable change $\mathbf{r}=\mathbf{x}-\mathbf{z}$, thus providing integrals (12).

In [18] it has been proved that for $\forall i \in \mathbf{N}_{0}$ it holds:

$$
\begin{aligned}
\int_{x_{1}-\varepsilon}^{x_{1}+\varepsilon} & \mathbf{G}_{p p}(\mathbf{r} ; \mathbf{n}(\mathbf{x}) ; \mathbf{l}(\mathbf{y})) r_{1}^{i} \mathrm{~d} r_{1}= \\
& \log \|\mathbf{r}\| \mathbf{L}_{p p}^{(i)}+\arctan \left(\frac{r_{1}}{x_{2}}\right) \mathbf{A}_{p p}^{(i)}+\frac{1}{r^{2}} \mathbf{S}_{p p}^{(i)}+\left.\frac{1}{r^{4}} \mathbf{H}_{p p}^{(i)}\right|_{r_{1}=x_{1}-\varepsilon} ^{r_{1}=x_{1}+\varepsilon}
\end{aligned}
$$

where $\mathbf{L}_{p p}^{(i)}, \mathbf{A}_{p p}^{(i)}, \mathbf{S}_{p p}^{(i)}, \mathbf{H}_{p p}^{(i)}$ are suitable matrices. By moving point $\mathbf{x}$ to the boundary, $\Omega \ni \mathbf{x} \rightarrow \mathbf{x}_{0} \in \Gamma_{\varepsilon}$, it is straightforward to get:

$$
\begin{aligned}
& \mathbf{L}_{p p}^{(0)}=\mathbf{A}_{p p}^{(0)}=\lim _{\mathbf{x} \rightarrow \mathbf{x}_{0}} \mathbf{H}_{p p}^{(0)} \frac{1}{r^{4}}=\mathbf{0}, \\
& \left.\lim _{\mathbf{x} \rightarrow \mathbf{x}_{0}} \frac{1}{r^{2}} \mathbf{S}_{p p}^{(0)}\right|_{r_{1}=-\varepsilon} ^{r_{1}=+\varepsilon}=-\left.\frac{1}{2 \pi} \frac{G}{1-\nu}\left(\begin{array}{cc}
n_{2} & n_{1} \\
n_{1} & n_{2}
\end{array}\right) \frac{1}{r_{1}}\right|_{r_{1}=-\varepsilon} ^{r_{1}=\varepsilon} \\
& \lim _{\mathbf{x} \rightarrow \mathbf{x}_{0}} \mathbf{S}_{p p}^{(1)} \frac{1}{r^{2}}=\lim _{\mathbf{x} \rightarrow \mathbf{x}_{0}} \mathbf{H}_{p p}^{(1)} \frac{1}{r^{4}}=\mathbf{0}, \quad \lim _{\mathbf{x} \rightarrow \mathbf{x}_{0}} \mathbf{L}_{p p}^{(1)}=\frac{1}{2 \pi} \frac{G}{1-\nu}\left(\begin{array}{cc}
n_{2} & n_{1} \\
n_{1} & n_{2}
\end{array}\right)
\end{aligned}
$$

and:

$$
\left.\lim _{\mathbf{x} \rightarrow \mathbf{x}_{0}} \arctan \left(\frac{r_{1}}{x_{2}}\right) \mathbf{A}_{p p}^{(1)}\right|_{r_{1}=-\varepsilon} ^{r_{1}=+\varepsilon}=\frac{G}{1-\nu}\left(\begin{array}{cc}
n_{1} & 0 \\
0 & 0
\end{array}\right)
$$

All singular terms cancel out in the limit process, without recourse to any apriori interpretation in the Hadamard's finite part (HFP) sense [5], as mentioned 
by various authors [9], [21]. However, there exists an intimate relationship [22] between HBIEs and HFP ${ }^{4}$, provided by the following proposition

Proposition 2.2 For any $\varepsilon \in \mathbf{R}$ :

$$
\begin{aligned}
\lim _{\mathbf{x} \rightarrow \mathbf{0}} & \int_{x_{1}-\varepsilon}^{x_{1}+\varepsilon} \mathbf{G}_{p p}(\mathbf{r} ; \mathbf{n}(\mathbf{x}) ; \mathbf{l}(\mathbf{y})) \mathrm{d} r_{1}=f_{-\varepsilon}^{\varepsilon} \mathbf{G}_{p p}(-\mathbf{y} ; \mathbf{n}(\mathbf{0}) ; \mathbf{l}(\mathbf{y})) \mathrm{d} r_{1} \\
\lim _{\mathbf{x} \rightarrow \mathbf{0}} & \int_{x_{1}-\varepsilon}^{x_{1}+\varepsilon} \mathbf{G}_{p p}(\mathbf{r} ; \mathbf{n}(\mathbf{x}) ; \mathbf{l}(\mathbf{y})) r_{1} \mathrm{~d} r_{1}= \\
\quad & \quad_{-\varepsilon}^{\varepsilon} \mathbf{G}_{p p}(-\mathbf{y} ; \mathbf{n}(\mathbf{0}) ; \mathbf{l}(\mathbf{y})) r_{1} \mathrm{~d} r_{1}+\frac{G}{1-\nu}\left(\begin{array}{cc}
n_{1} & 0 \\
0 & 0
\end{array}\right)
\end{aligned}
$$

Proof: $\quad$ Straightforward passages permit to obtain in the local reference $\mathcal{L}$ (see also [18]):

$$
\begin{aligned}
& f_{-\varepsilon}^{\varepsilon} \mathbf{G}_{p p}(-\mathbf{y} ; \mathbf{n}(\mathbf{0}) ; \mathbf{l}(\mathbf{y})) \mathrm{d} r_{1}=-\frac{1}{\pi} \frac{G}{1-\nu}\left(\begin{array}{cc}
n_{2} & n_{1} \\
n_{1} & n_{2}
\end{array}\right) \frac{1}{\varepsilon} \\
& f_{-\varepsilon}^{\varepsilon} \mathbf{G}_{p p}(-\mathbf{y} ; \mathbf{n}(\mathbf{0}) ; \mathbf{l}(\mathbf{y})) r_{1} \mathrm{~d} r_{1}=\left.\frac{1}{2 \pi} \frac{G}{1-\nu}\left(\begin{array}{cc}
n_{2} & n_{1} \\
n_{1} & n_{2}
\end{array}\right) \log \|\mathbf{r}\|\right|_{r_{1}=-\varepsilon} ^{r_{1}=\varepsilon}=0
\end{aligned}
$$

The thesis follows by comparison with eq. (13)

Corollary 2.2.1 It holds in $\mathcal{L}$ :

$$
\begin{gathered}
\lim _{\mathbf{x} \rightarrow \mathbf{0}} \int_{\Gamma_{\varepsilon}} \mathbf{G}_{p p}(\mathbf{x}-\mathbf{y} ; \mathbf{n}(\mathbf{x}), \mathbf{l}(\mathbf{y})) \mathrm{d} \Gamma_{\mathbf{y}}=f_{\Gamma_{\varepsilon}} \mathbf{G}_{p p}(-\mathbf{y} ; \mathbf{n}(\mathbf{0}), \mathbf{l}(\mathbf{y})) \mathrm{d} \Gamma_{\mathbf{y}} \\
\lim _{\mathbf{x} \rightarrow \mathbf{0}} \int_{\Gamma_{\varepsilon}} \mathbf{G}_{p p}(\mathbf{x}-\mathbf{y} ; \mathbf{n}(\mathbf{x}), \mathbf{l}(\mathbf{y})) \boldsymbol{\epsilon}(\mathbf{0}) \mathbf{y} \mathrm{d} \Gamma_{\mathbf{y}}=f_{\Gamma_{\varepsilon}} \mathbf{G}_{p p}(-\mathbf{y} ; \mathbf{n}(\mathbf{0}), \mathbf{l}(\mathbf{y})) \boldsymbol{\epsilon}(\mathbf{0}) \mathbf{y} \mathrm{d} \Gamma_{\mathbf{y}}+ \\
\quad-\frac{G}{1-\nu}\left(\begin{array}{cr}
n_{1} & 0 \\
0 & 0
\end{array}\right) \boldsymbol{\epsilon}(\mathbf{0}) \mathbf{e}_{1}
\end{gathered}
$$

The following term, due to the limit (13):

$$
\mathbf{D}_{p p}\left(\mathbf{x}_{0}, \mathbf{n}\left(\mathbf{x}_{0}\right)\right) \stackrel{\text { def }}{=}-\frac{G}{1-\nu}\left(\begin{array}{cc}
n_{1} & 0 \\
0 & 0
\end{array}\right) \boldsymbol{\epsilon}\left(\mathbf{x}_{0}\right)\left(\mathbf{e}_{1} \otimes \mathbf{p}\left(\mathbf{x}_{0}, \mathbf{l}\left(\mathbf{x}_{0}\right)\right)\right) \frac{1}{\left\|\mathbf{p}\left(\mathbf{x}_{0}, \mathbf{l}\left(\mathbf{x}_{0}\right)\right)\right\|^{2}}
$$

\footnotetext{
${ }^{4}$ It has already been proved (see [4] and [23] among others) that the hypersingular integral in (1) can be interpreted as a HFP in the limit as an internal field point $\mathbf{x}$ approaches the boundary. In [24], the same conclusion has been obtained by an alternate definition of HFP, without the need for a limiting process. Making recourse to the distribution theory [6], the dual BIEs are obtained by the application of a trace operator to the representation formulae (1). In such an approach, the strongly singular and hypersingular integrals can be expressed by means of discontinuity jumps (also called "free terms") of these integrals on the boundary summed with the values of the integrals on the boundary existing only in the sense of Cauchy Principal Value (CPV) or in the sense of the HFP. By exploiting Green's functions properties, the commutativity of the two operators of traction and trace has also been proved, showing the consistency of all different approaches of derivations of the traction BIE.
} 
plays the role of a free-term, more precisely it is the contribution of the hypersingular kernel to the free-term $\mathbf{D}(\mathbf{x})$ in equation (2). It is evident that $\mathbf{D}_{p p}$ depends on the elastic properties of the body and on the selected direction $\mathbf{n}\left(\mathbf{x}_{0}\right)$ at point $\mathbf{x}_{0} \in \Gamma_{\varepsilon}$ : when it is taken as the outward normal at $\mathbf{x}_{0}$, that is $\mathbf{n}\left(\mathbf{x}_{0}\right)=\mathbf{e}_{2}$, then $\mathbf{D}_{p p}$ vanishes and, as a common belief, the free term $\mathbf{D}(\mathbf{x})$ is solely due to the strongly singular kernel. On the contrary, when the traction is sought with respect to a direction $\mathbf{n}\left(\mathbf{x}_{0}\right)$ which differs from the normal at $\mathbf{x}_{0}$, then the hypersingular kernel contributes to the free term even on smooth boundaries.

Contributions by the hypersingular kernel have been observed in literature in [9], [13] for the hypersingular boundary integral equation for displacement derivatives and in [8], [16] in the framework of potential theory, when the boundary curvature and the tangent vector to the boundary are not smooth. Free term (17) has a very different nature.

Proposition 2.2 states that the HFP is the outcome of a limit process for the hypersingular kernel on every smooth boundary. Therefore, on every smooth boundary, HFP in equation (2) is nothing but a consequence of a property of the hypersingular kernel.

\subsection{Almost everywhere $C^{2}$ boundaries}

Let $\tilde{\mathbf{y}}(s)=\left\{\tilde{y}_{1}(s), \tilde{y}_{2}(s)\right\}, 0 \leq s \leq l$ be the parametric equations of curve $\Gamma_{\varepsilon}$ with $s$ denoting the curvilinear abscissa; assume $\tilde{\mathbf{y}}(s) \in C^{2}[0, l]$ with exception of a single point $s_{0}$, and $\tilde{\mathbf{y}}(s) \in C^{1}[0, l]$. Define $\Gamma_{\varepsilon}^{-} \subset \Gamma_{\varepsilon}$ the curve parameterized by $\tilde{\mathbf{y}}(s)=\left\{\tilde{y}_{1}(s), \tilde{y}_{2}(s)\right\}, 0 \leq s \leq s_{0}$ and $\Gamma_{\varepsilon}^{+} \stackrel{\text { def }}{=} \Gamma_{\varepsilon}-\Gamma_{\varepsilon}^{-}$its complementary part described by $\tilde{\mathbf{y}}(s)=\left\{\tilde{y}_{1}(s), \tilde{y}_{2}(s)\right\}, s_{0} \leq s \leq l$. A local coordinate system $\mathcal{L}$, centered at $\mathbf{x}_{0} \in \Gamma_{\varepsilon}$, is considered with axis $y_{1}, y_{2}$ tangent and normal to $\Gamma_{\varepsilon}$ at $\mathbf{x}_{0}$, respectively (see figure 2). Point $\mathbf{x}_{0}$ is selected such that the parametric equations of $\Gamma_{\varepsilon} \operatorname{read} \mathbf{y}\left(y_{1}^{+}\right)=\left\{y_{1}^{+}, y_{2}^{+}\left(y_{1}^{+}\right)\right\}, y_{1}^{+} \in I_{\varepsilon}^{+} \stackrel{\text { def }}{=}[0, \varepsilon]$ and $\mathbf{y}\left(y_{1}^{-}\right)=$ $\left\{y_{1}^{-}, y_{2}^{-}\left(y_{1}^{-}\right)\right\}, y_{1}^{-} \in I_{\varepsilon}^{-} \stackrel{\text { def }}{=}[-\varepsilon, 0]$ in the local coordinate system $\mathcal{L}$.

In view of the given definition of $\Gamma_{\varepsilon}$, the limit (9) is here considered also when $y^{\prime \prime}(\cdot)$ is not defined in a finite number of points on $\Gamma^{5}$. It is worth rewriting the limit (9) as follows $(\mathbf{x} \in \Omega)$ :

$$
\begin{aligned}
& \lim _{\mathbf{x} \rightarrow \mathbf{x}_{0}}\left\{\int_{\Gamma_{\varepsilon}^{-}} \mathbf{G}_{p p}(\mathbf{r} ; \mathbf{n}(\mathbf{x}), \mathbf{l}(\mathbf{y})) \mathrm{d} \Gamma_{\mathbf{y}} \mathbf{u}\left(\mathbf{x}_{0}\right)+\int_{\Gamma_{\varepsilon}^{-}} \mathbf{G}_{p p}(\mathbf{r} ; \mathbf{n}(\mathbf{x}), \mathbf{l}(\mathbf{y})) \boldsymbol{\epsilon}\left(\mathbf{x}_{0}\right) \mathbf{y} \mathrm{d} \Gamma_{\mathbf{y}}+\right. \\
&\left.\int_{\Gamma_{\varepsilon}^{+}} \mathbf{G}_{p p}(\mathbf{r} ; \mathbf{n}(\mathbf{x}), \mathbf{l}(\mathbf{y})) \mathrm{d} \Gamma_{\mathbf{y}} \mathbf{u}\left(\mathbf{x}_{0}\right)+\int_{\Gamma_{\varepsilon}^{+}} \mathbf{G}_{p p}(\mathbf{r} ; \mathbf{n}(\mathbf{x}), \mathbf{l}(\mathbf{y})) \boldsymbol{\epsilon}\left(\mathbf{x}_{0}\right) \mathbf{y} \mathrm{d} \Gamma_{\mathbf{y}}\right\}
\end{aligned}
$$

The free term analysis that follows pertains to $\Gamma_{\varepsilon}^{+}$, but results may be trivially generalized to the whole $\Gamma_{\varepsilon}$. It will be assumed that:

Hypotheses 2.2 Let $\Gamma_{\varepsilon}^{+}$be defined by $y_{2}\left(y_{1}\right)=y_{1}^{\alpha}, \alpha \geq 2$

\footnotetext{
${ }^{5}$ As an example of such a curve, consider a straight line $y_{2}^{-}=0$ joined with an arc of parabola $y_{2}^{+}=\left(y_{1}^{+}\right)^{2}$ : it will be called the "prototype curve".
} 
For being $y_{2}^{\prime}(0)=0$ by definition, all results based on hypothesis 2.2 hold also for all $y_{2}(\cdot) \in C^{2}(0, \varepsilon]$ that admit a Taylor expansion around $y_{1}=0$. It is trivial to see that the regularity requirements are much lower than hypothesis 2.1 ; as a main consequence, all lemmas in the previous section fail and it is not possible to reduce the hypersingular integral from $\Gamma_{\varepsilon}$ to $I_{\varepsilon}$ without introducing a free term. It is easy to prove, for instance, that equation (10) is no longer valid for the "prototype curve".

Lemma 2.3 If $y_{2}\left(y_{1}\right)=y_{1}^{2}$ then two functions $\mathbf{g}_{0}\left(\mathbf{x} ; y_{1}\right) \in C^{0}\left(B_{\tilde{\varepsilon}} \times \Gamma_{\varepsilon}\right)$ and $\mathbf{g}_{-1}\left(\mathbf{x} ; y_{1}\right)$ exist such that:

$\mathbf{G}_{p p}(\mathbf{x}-\mathbf{y} ; \mathbf{n}(\mathbf{x}), \mathbf{l}(\mathbf{y})) \sqrt{1+y_{2}^{\prime}\left(y_{1}\right)^{2}}=\mathbf{G}_{p p}(\mathbf{x}-\mathbf{z} ; \mathbf{n}(\mathbf{x}), \mathbf{l}(\mathbf{z}))+\mathbf{g}_{0}\left(\mathbf{x} ; y_{1}\right)+\mathbf{g}_{-1}\left(\mathbf{x} ; y_{1}\right)$

with $\mathbf{g}_{-1}\left(\mathbf{0} ; y_{1}\right)=\mathbf{0} \quad \forall y_{1} \in I_{\varepsilon} \backslash 0$.

Proof: $\quad$ Consider $\mathbf{g}_{-1}\left(\mathbf{x} ; y_{1}\right)$ as in appendix 1. By direct substitution $\mathbf{g}_{-1}\left(\mathbf{0} ; y_{1}\right)=\mathbf{0}$ when $y_{1} \neq 0$. Furthermore, the function

$$
\mathbf{g}_{0}\left(\mathbf{x} ; y_{1}\right) \stackrel{\text { def }}{=} \mathbf{G}_{p p}(\mathbf{x}-\mathbf{y} ; \mathbf{n}(\mathbf{x}), \mathbf{l}(\mathbf{y})) \sqrt{1+y_{2}^{\prime}\left(y_{1}\right)^{2}}-\mathbf{G}_{p p}(\mathbf{x}-\mathbf{z} ; \mathbf{n}(\mathbf{x}), \mathbf{l}(\mathbf{z}))-\mathbf{g}_{-1}\left(\mathbf{x} ; y_{1}\right)
$$

shows to be continuous in $B_{\tilde{\varepsilon}} \times \Gamma_{\varepsilon}^{+}$.

Corollary 2.3.1 If $\mathrm{x} \in \Omega$, it holds:

$$
\begin{aligned}
& \int_{\Gamma_{\varepsilon}^{+}} \mathbf{G}_{p p}(\mathbf{x}-\mathbf{y} ; \mathbf{n}(\mathbf{x}), \mathbf{l}(\mathbf{y})) \mathrm{d} \Gamma_{\mathbf{y}}= \\
& \quad \int_{I_{\varepsilon}^{+}} \mathbf{G}_{p p}(\mathbf{x}-\mathbf{z} ; \mathbf{n}(\mathbf{x}), \mathbf{l}(\mathbf{z})) \mathrm{d} y_{1}+\int_{I_{\varepsilon}^{+}} \mathbf{g}_{-1}\left(\mathbf{x} ; y_{1}\right) \mathrm{d} y_{1}+O(\varepsilon)
\end{aligned}
$$

Lemma 2.3 permits, similarly to lemmas 2.1 and 2.2 , to "move" the hypersingular integrals from $\Gamma_{\varepsilon}^{+}$to $I_{\varepsilon}^{+}$. The induced approximation is stated by the following lemma:

Lemma 2.4 It holds:

$$
\lim _{\mathbf{x} \rightarrow \mathbf{0}} \int_{I_{\varepsilon}^{+}} \mathbf{g}_{-1}\left(\mathbf{x} ; y_{1}\right) \mathrm{d} y_{1}=\frac{G}{2 \pi(1-\nu)}\left(\begin{array}{cc}
-3 n_{1} & n_{2} \\
n_{2} & n_{1}
\end{array}\right)=-\lim _{\mathbf{x} \rightarrow \mathbf{0}} \int_{I_{\varepsilon}^{-}} \mathbf{g}_{-1}\left(\mathbf{x} ; y_{1}\right) \mathrm{d} y_{1}
$$

Proof: By direct integration of $\mathbf{g}_{-1}\left(\mathbf{x} ; y_{1}\right)$ as it is in appendix $1 .$. 
In view of lemma 2.4, the approximation of integral (18) on $I_{\varepsilon}^{+}$is no longer $O(\varepsilon)$, as in lemmas 2.1 and 2.2, but $O(1)$. The r.h.s. of equation (20) acts as a free term $^{6}$ : it depends on the material parameters $G$ and $\nu$ as well as on the selected direction $\mathbf{n}(\mathbf{x})$. Lemma 2.4 permits to prove the following propositions.

Proposition 2.3 On every $C^{2}$ boundary $\Gamma_{\varepsilon}$ it holds:

$$
\lim _{\mathbf{x} \rightarrow \mathbf{0}} \int_{\Gamma_{\varepsilon}} \mathbf{G}_{p p}(\mathbf{x}-\mathbf{y} ; \mathbf{n}(\mathbf{x}), \mathbf{l}(\mathbf{y})) \mathrm{d} \Gamma_{\mathbf{y}}=\lim _{\mathbf{x} \rightarrow \mathbf{0}} \int_{I_{\varepsilon}} \mathbf{G}_{p p}(\mathbf{x}-\mathbf{z} ; \mathbf{n}(\mathbf{x}), \mathbf{l}(\mathbf{z})) \mathrm{d} y_{1}+O(\varepsilon)
$$

As a consequence of lemma 2.3, results in section 2.1 apply also to $C^{2}$ boundaries, because free terms arising in boundary approximation cancel themselves out in the sum. The proposition 2.1 extends as follows:

Proposition 2.4 For solving integral (18) on almost everywhere $C^{2}$-boundaries it is sufficient to solve integrals:

$$
\int_{x_{1}-\varepsilon}^{x_{1}} \mathbf{G}_{p p}(\mathbf{r} ; \mathbf{n}(\mathbf{x}) ; \mathbf{l}(\mathbf{z})) r_{1}^{i} \mathrm{~d} r_{1} \quad i=0,1 \quad \mathbf{x} \in \Omega
$$

Proof: Apply the proof of proposition 2.1 to $I_{\varepsilon}^{+}$and $I_{\varepsilon}^{-}$.

In spite of their formal similarity, integral (21) is very different from integral (12). In fact point $\mathbf{x}$ is an extremity of integral (21) and limit to the boundary $\Omega \ni \mathbf{x} \rightarrow \mathbf{x}_{0} \in \Gamma$ is not expected to exist. Nevertheless, equation (14) extends as follows:

Proposition 2.5 For any $\varepsilon \in \mathbf{R}$ :

$$
\begin{gathered}
\lim _{\mathbf{x} \rightarrow \mathbf{0}}\left\{\int_{-\varepsilon}^{0} \mathbf{G}_{p p}(\mathbf{r} ; \mathbf{n}(\mathbf{x}) ; \mathbf{l}(\mathbf{z})) \mathrm{d} y_{1}+\int_{0}^{\varepsilon} \mathbf{G}_{p p}(\mathbf{r} ; \mathbf{n}(\mathbf{x}) ; \mathbf{l}(\mathbf{z})) \mathrm{d} y_{1}\right\}= \\
\text { f }_{I_{\varepsilon}} \mathbf{G}_{p p}(\mathbf{r} ; \mathbf{n}(\mathbf{0}) ; \mathbf{l}(\mathbf{z})) \mathrm{d} y_{1}
\end{gathered}
$$

Proof: $\quad$ With reference to figure 2 the integral $(\mathrm{x} \in \Omega)$

$$
\int_{0}^{\varepsilon} \mathbf{G}_{p p}(\mathbf{r} ; \mathbf{n}(\mathbf{x}) ; \mathbf{l}(\mathbf{z})) \mathrm{d} y_{1}=\int_{x_{1}-\varepsilon}^{x_{1}} \mathbf{G}_{p p}(\mathbf{r} ; \mathbf{n}(\mathbf{x}) ; \mathbf{l}(\mathbf{z})) \mathrm{d} r_{1}=\frac{1}{r^{2}} \mathbf{S}_{p p}^{(0)}+\left.\frac{1}{r^{4}} \mathbf{H}_{p p}^{(0)}\right|_{r_{1}=x_{1}-\varepsilon} ^{r_{1}=x_{1}}
$$

has been solved in [18] by means of the variable change $\mathbf{r}=\mathbf{x}-\mathbf{y}$; matrices $\mathbf{S}_{p p}^{(0)}, \mathbf{H}_{p p}^{(0)}$ follow in the local reference $\mathcal{L}$ :

$$
\begin{aligned}
\mathbf{S}_{p p}^{(0)} & =\frac{G}{2 \pi(\nu-1)}\left(\begin{array}{cc}
n_{2} r_{1}-3 n_{1} r_{2} & n_{1} r_{1}+n_{2} r_{2} \\
n_{1} r_{1}+n_{2} r_{2} & n_{2} r_{1}+n_{1} r_{2}
\end{array}\right) \\
\mathbf{H}_{p p}^{(0)} & =\frac{G r_{2}^{2}}{\pi(1-\nu)}\left(\begin{array}{cc}
n_{2} r_{1}-n_{1} r_{2} & n_{1} r_{1}+n_{2} r_{2} \\
n_{1} r_{1}+n_{2} r_{2} & n_{1} r_{2}-n_{2} r_{1}
\end{array}\right)
\end{aligned}
$$

\footnotetext{
${ }^{6}$ it has the same origin of the hypersingular free term in [13], but it refers to the kernel $\mathbf{G}_{p p}$
} 
A similar result holds for the integral $(\mathrm{x} \in \Omega)$

$$
\int_{-\varepsilon}^{0} \mathbf{G}_{p p}(\mathbf{r} ; \mathbf{n}(\mathbf{x}) ; \mathbf{l}(\mathbf{z})) \mathrm{d} y_{1}=\int_{x_{1}}^{x_{1}+\varepsilon} \mathbf{G}_{p p}(\mathbf{r} ; \mathbf{n}(\mathbf{x}) ; \mathbf{l}(\mathbf{z})) \mathrm{d} r_{1}=\frac{1}{r^{2}} \mathbf{S}_{p p}^{(0)}+\left.\frac{1}{r^{4}} \mathbf{H}_{p p}^{(0)}\right|_{r_{1}=x_{1}} ^{r_{1}=x_{1}+\varepsilon}
$$

The limit to the boundary $\Omega \ni \mathbf{x} \rightarrow \mathbf{x}_{0} \in \Gamma$ is taken by means of the norm $\|\mathbf{x}\| \rightarrow 0$ in $\mathcal{L}$ and of a direction, selected through the angle $\theta$ as in figure 3.

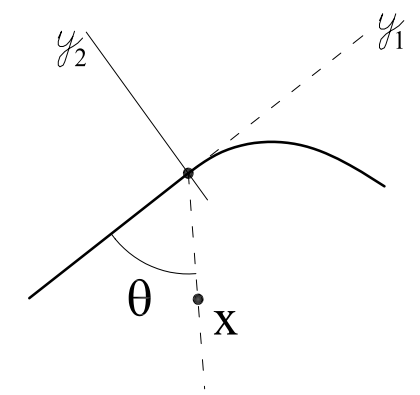

Figure 3: Limit process $\Omega \ni \mathbf{x} \rightarrow \mathbf{x}_{0} \in \Gamma$.

It is worth noting that the limit

$$
\lim _{\mathbf{x} \rightarrow \mathbf{0}} \int_{0}^{\varepsilon} \mathbf{G}_{p p}(\mathbf{r} ; \mathbf{n}(\mathbf{x}) ; \mathbf{l}(\mathbf{z})) \mathrm{d} y_{1}=\oint_{0}^{\varepsilon} \mathbf{G}_{p p}(\mathbf{r} ; \mathbf{n}(\mathbf{0}) ; \mathbf{l}(\mathbf{z})) \mathrm{d} y_{1}+\frac{G}{2 \pi(1-\nu)} \lim _{\mathbf{x} \rightarrow \mathbf{0}} \frac{\mathbf{W}(\theta)}{\|\mathbf{x}\|}
$$

with:

$\mathbf{W}(\theta) \stackrel{\text { def }}{=}\left(\begin{array}{cc}\frac{n_{2}(\cos (\theta)+\cos (3 \theta))-n_{1}(3 \sin (\theta)+\sin (3 \theta))}{2} & \cos (2 \theta)\left(n_{1} \cos (\theta)+n_{2} \sin (\theta)\right) \\ \cos (2 \theta)\left(n_{1} \cos (\theta)+n_{2} \sin (\theta)\right) & \frac{3 n_{2}(\cos (\theta)-\cos (3 \theta))+n_{1}(-\sin (\theta)+\sin (3 \theta))}{2}\end{array}\right)$

is not well defined. All "inconsistent" terms however cancel out in the sum (22), so that the thesis is proved.

As a consequence, no free terms arise from the constant hypersingular integral on $I_{\varepsilon}$, similarly to (14). Furthermore, even in the presence of an almost $C^{2}$ boundary, the finite part of Hadamard concept is the outcome of a limit process for the hypersingular kernel when applied to a constant term. In view of lemma 2.3 and of proposition 2.5 , it is straightforward to prove that:

Proposition 2.6 If $y_{2}^{ \pm}\left(y_{1}\right) \sim \alpha^{ \pm} y_{1}^{2}$ then

$$
\begin{aligned}
\lim _{\mathbf{x} \rightarrow \mathbf{0}} \int_{\Gamma_{\varepsilon}} \mathbf{G}_{p p}(\mathbf{x}-\mathbf{y} ; \mathbf{n}(\mathbf{x}), \mathbf{l}(\mathbf{y})) \mathrm{d} \Gamma_{\mathbf{y}}= \\
\quad \quad \quad_{\Gamma_{\varepsilon}} \mathbf{G}_{p p}(-\mathbf{y} ; \mathbf{n}(\mathbf{0}), \mathbf{l}(\mathbf{y})) \mathrm{d} \Gamma_{\mathbf{y}}+\frac{G\left(\alpha^{+}-\alpha^{-}\right)}{2 \pi(1-\nu)}\left(\begin{array}{cc}
-3 n_{1} & n_{2} \\
n_{2} & n_{1}
\end{array}\right)
\end{aligned}
$$


Proof: From lemma 2.3 it comes out:

$$
\begin{aligned}
\lim _{\mathbf{x} \rightarrow \mathbf{0}} \int_{\Gamma_{\varepsilon}} \mathbf{G}_{p p}(\mathbf{x}-\mathbf{y} ; \mathbf{n}(\mathbf{x}), \mathbf{l}(\mathbf{y})) \mathrm{d} \Gamma_{\mathbf{y}}= \\
\quad \lim _{\mathbf{x} \rightarrow \mathbf{0}}\left\{\int_{I_{\varepsilon}^{-}} \mathbf{g}_{-1}\left(\mathbf{x} ; y_{1}\right) \mathrm{d} y_{1}+\int_{I_{\varepsilon}^{+}} \mathbf{g}_{-1}\left(\mathbf{x} ; y_{1}\right) \mathrm{d} y_{1}\right\}+ \\
+\lim _{\mathbf{x} \rightarrow \mathbf{0}}\left\{\int_{I_{\varepsilon}^{-}} \mathbf{g}_{0}\left(\mathbf{x} ; y_{1}\right) \mathrm{d} y_{1}+\int_{I_{\varepsilon}^{+}} \mathbf{g}_{0}\left(\mathbf{x} ; y_{1}\right) \mathrm{d} y_{1}\right\}+ \\
+\lim _{\mathbf{x} \rightarrow \mathbf{0}}\left\{\int_{I_{\varepsilon}^{-}} \mathbf{G}_{p p}(\mathbf{x}-\mathbf{z} ; \mathbf{n}(\mathbf{x}), \mathbf{l}(\mathbf{z})) \mathrm{d} y_{1}+\int_{I_{\varepsilon}^{+}} \mathbf{G}_{p p}(\mathbf{x}-\mathbf{z} ; \mathbf{n}(\mathbf{x}), \mathbf{l}(\mathbf{z})) \mathrm{d} y_{1}\right\}
\end{aligned}
$$

by proposition 2.5:

$$
\begin{aligned}
& =\frac{G\left(\alpha^{+}-\alpha^{-}\right)}{2 \pi(1-\nu)}\left(\begin{array}{cc}
-3 n_{1} & n_{2} \\
n_{2} & n_{1}
\end{array}\right)+\lim _{\mathbf{x} \rightarrow \mathbf{0}}\left\{\int_{I_{\varepsilon}^{-}} \mathbf{g}_{0}\left(\mathbf{x} ; y_{1}\right) \mathrm{d} y_{1}+\int_{I_{\varepsilon}^{+}} \mathbf{g}_{0}\left(\mathbf{x} ; y_{1}\right) \mathrm{d} y_{1}\right\}+ \\
& \quad+f_{I_{\varepsilon}} \mathbf{G}_{p p}(-\mathbf{y} ; \mathbf{n}(\mathbf{0}), \mathbf{l}(\mathbf{y})) \mathrm{d} \Gamma_{\mathbf{y}}
\end{aligned}
$$

and the thesis follows by noting that:

$$
\begin{aligned}
& f \mathbf{G}_{\Gamma_{\varepsilon}}(-\mathbf{y} ; \mathbf{n}(\mathbf{0}), \mathbf{l}(\mathbf{y})) \mathrm{d} \Gamma_{\mathbf{y}}= \\
& \quad \lim _{\mathbf{x} \rightarrow \mathbf{0}}\left\{\int_{I_{\varepsilon}^{-}} \mathbf{g}_{0}\left(\mathbf{x} ; y_{1}\right) \mathrm{d} y_{1}+\int_{I_{\varepsilon}^{+}} \mathbf{g}_{0}\left(\mathbf{x} ; y_{1}\right) \mathrm{d} y_{1}\right\}+f_{I_{\varepsilon}} \mathbf{G}_{p p}(-\mathbf{y} ; \mathbf{n}(\mathbf{0}), \mathbf{l}(\mathbf{y})) \mathrm{d} \Gamma_{\mathbf{y}}
\end{aligned}
$$

Identity (15) extends as follows:

Proposition 2.7 For any $\varepsilon \in \mathbf{R}$ :

$$
\begin{aligned}
\lim _{\mathbf{x} \rightarrow \mathbf{0}}\{ & \left.\int_{x_{1}-\varepsilon}^{x_{1}} \mathbf{G}_{p p}(\mathbf{r} ; \mathbf{n}(\mathbf{x}) ; \mathbf{l}(\mathbf{z})) r_{1} \mathrm{~d} r_{1}+\int_{x_{1}}^{x_{1}+\varepsilon} \mathbf{G}_{p p}(\mathbf{r} ; \mathbf{n}(\mathbf{x}) ; \mathbf{l}(\mathbf{z})) r_{1} \mathrm{~d} r_{1}\right\}= \\
& f_{-\varepsilon}^{\varepsilon} \mathbf{G}_{p p}(\mathbf{r} ; \mathbf{n}(\mathbf{0}) ; \mathbf{l}(\mathbf{z})) r_{1} \mathrm{~d} r_{1}+\frac{G}{1-\nu}\left(\begin{array}{cc}
n_{1} & 0 \\
0 & 0
\end{array}\right)
\end{aligned}
$$

Proof: The thesis is proved by direct substitution, observing again that "inconsistent" terms cancel out in the sum, with the same arguments of proposition 2.5. See also proposition .... with $\varphi=0$ in the companion paper [19].

Similarly, identity (16) holds as it stands on almost every $C^{2}$ boundaries.

The term of equation (17) is again the contribution of the hypersingular kernel to the free-term $\mathbf{D}(\mathbf{x})$ in equation (2) for every almost everywhere $C^{2}$ boundary. It is worth noting that such a free term does not depend on the regularity of the 
boundary itself, but is merely due to the choice of a direction $\mathbf{n}(\mathbf{x})$ different from the boundary normal $\mathbf{l}(\mathbf{x})$. Free term (20) has a very different nature from (17), because it is only due to the (lack of) smoothness of the boundary $\Gamma$.

Furthermore, propositions 2.5 and 2.7 state that the HFP is the outcome of a limit process for the hypersingular kernel on every almost everywhere $C^{2}$ boundary. Therefore, HFP in equation (2) is nothing but a consequence of a property of the hypersingular kernel.

\section{$2.3 \quad C^{1}$ boundaries}

Let $\tilde{\mathbf{y}}(s)=\left\{\tilde{y}_{1}(s), \tilde{y}_{2}(s)\right\}, 0 \leq s \leq l$ be the parametric equations of curve $\Gamma_{\varepsilon}$ with $s$ denoting the curvilinear abscissa; assume $\tilde{\mathbf{y}}(\cdot)$ differentiable in $0 \leq s \leq l$. Consider a local coordinate system $\mathcal{L}$ centered at $\mathbf{x}_{0} \in \Gamma_{\varepsilon}$, as in figure 2-a, with axis $y_{1}, y_{2}$ tangent and normal to $\Gamma_{\varepsilon}$ at $\mathbf{x}_{0}$, respectively. Point $\mathbf{x}_{0}$ is selected such that the parametric equations of $\Gamma_{\varepsilon}$ with respect to $y_{1}$ read $\mathbf{y}\left(y_{1}\right)=\left\{y_{1}, y_{2}\left(y_{1}\right)\right\}, y_{1} \in$ $I_{\varepsilon} \stackrel{\text { def }}{=}[-\varepsilon, \varepsilon]$. Assume further that $y_{2}\left(y_{1}\right) \in C^{1}\left(I_{\varepsilon}\right)$.

By having reduced the regularity conditions on $\Gamma_{\varepsilon}$ with respect to hypothesis 2.2 , all lemmas in the previous section fail and it is not possible to reduce the hypersingular integral from $\Gamma_{\varepsilon}$ to $I_{\varepsilon}$ by means of an integrable function $\mathbf{g}_{-1}\left(\mathbf{x} ; y_{1}\right)$ on $I_{\varepsilon}$. Unfortunately, I am not able to prove propositions in what follows for the whole class of $C^{1}$ boundaries but only for boundaries $y_{2}\left(y_{1}\right)$ that have an asymptotic behavior $y_{2}\left(y_{1}\right) \sim y_{1}^{3 / 2}$. At any rate, it can be conjectured that at least all boundaries having an asymptotic behavior $y_{2}\left(y_{1}\right) \sim y_{1}^{\alpha}$ with $3 / 2 \leq \alpha<2$ share the following basic properties; moreover, even this simple case leads to remarkable conclusions.

Lemma 2.5 If $y_{2}\left(y_{1}\right)=y_{1}^{3 / 2}$ then functions $\mathbf{g}_{0}\left(\mathbf{x} ; y_{1}\right) \in C^{0}\left(B_{\tilde{\varepsilon}} \times \Gamma_{\varepsilon}\right), \mathbf{g}_{-3 / 2}\left(\mathbf{x} ; y_{1}\right)$, $\mathbf{g}_{-1}\left(\mathbf{x} ; y_{1}\right)$, and $\mathbf{g}_{-1 / 2}\left(\mathbf{x} ; y_{1}\right)$ exist such that:

$$
\begin{aligned}
\mathbf{G}_{p p}(\mathbf{x}-\mathbf{y} ; \mathbf{n}(\mathbf{x}), \mathbf{l}(\mathbf{y})) \sqrt{1+y_{2}^{\prime}\left(y_{1}\right)^{2}}=\mathbf{G}_{p p}(\mathbf{x}-\mathbf{z} ; \mathbf{n}(\mathbf{x}), \mathbf{l}(\mathbf{z}))+ \\
\mathbf{g}_{-3 / 2}\left(\mathbf{x} ; y_{1}\right)+\mathbf{g}_{-1}\left(\mathbf{x} ; y_{1}\right)+\mathbf{g}_{-1 / 2}\left(\mathbf{x} ; y_{1}\right)+\mathbf{g}_{0}\left(\mathbf{x} ; y_{1}\right)
\end{aligned}
$$

with $\mathbf{g}_{i}\left(\mathbf{x} ; y_{1}\right) \sim O\left(y_{1}^{i}\right)$ for $i=-3 / 2,-1,-1 / 2$.

Proof: Consider $\mathbf{g}_{i}\left(\mathbf{x} ; y_{1}\right) \sim O\left(y_{1}^{i}\right)$ for $i=-3 / 2,-1,-1 / 2$ as in appendix 2. Furthermore, the function

$$
\begin{aligned}
\mathbf{g}_{0}\left(\mathbf{x} ; y_{1}\right) \stackrel{\text { def }}{=} \mathbf{G}_{p p}(\mathbf{x}-\mathbf{y} ; \mathbf{n}(\mathbf{x}), \mathbf{l}(\mathbf{y})) & \sqrt{1+y_{2}^{\prime}\left(y_{1}\right)^{2}}-\mathbf{G}_{p p}(\mathbf{x}-\mathbf{z} ; \mathbf{n}(\mathbf{x}), \mathbf{l}(\mathbf{z}))- \\
& \left(\mathbf{g}_{-3 / 2}\left(\mathbf{x} ; y_{1}\right)+\mathbf{g}_{-1}\left(\mathbf{x} ; y_{1}\right)+\mathbf{g}_{-1 / 2}\left(\mathbf{x} ; y_{1}\right)\right)
\end{aligned}
$$

shows to be continuous in $B_{\tilde{\varepsilon}} \times \Gamma_{\varepsilon}^{+}$. 
Corollary 2.5.1 If $\mathrm{x} \in \Omega$, it holds:

$$
\begin{aligned}
& \int_{\Gamma_{\varepsilon}^{+}} \mathbf{G}_{p p}(\mathbf{x}-\mathbf{y} ; \mathbf{n}(\mathbf{x}), \mathbf{l}(\mathbf{y})) \mathrm{d} \Gamma_{\mathbf{y}}=\int_{I_{\varepsilon}^{+}} \mathbf{G}_{p p}(\mathbf{x}-\mathbf{z} ; \mathbf{n}(\mathbf{x}), \mathbf{l}(\mathbf{z})) \mathrm{d} y_{1}+ \\
& \int_{I_{\varepsilon}^{+}} \mathbf{g}_{-3 / 2}\left(\mathbf{x} ; y_{1}\right) \mathrm{d} y_{1}+\int_{I_{\varepsilon}^{+}} \mathbf{g}_{-1}\left(\mathbf{x} ; y_{1}\right) \mathrm{d} y_{1}+\int_{I_{\varepsilon}^{+}} \mathbf{g}_{-1 / 2}\left(\mathbf{x} ; y_{1}\right) \mathrm{d} y_{1}+O(\varepsilon)
\end{aligned}
$$

Lemma 2.5 permits to "move" the hypersingular integrals from $\Gamma_{\varepsilon}^{+}$to $I_{\varepsilon}^{+}$. However, the function $\mathbf{g}_{-3 / 2}\left(\mathbf{x} ; y_{1}\right)$ is no longer integrable on $I_{\varepsilon}$ and the approximation is therefore questionable. The following lemma holds:

Lemma 2.6 It holds:

$$
\begin{gathered}
\lim _{\mathbf{x} \rightarrow \mathbf{0}} \int_{0}^{\varepsilon} \mathbf{g}_{-3 / 2}\left(\mathbf{x} ; y_{1}\right)+\mathbf{g}_{-1 / 2}\left(\mathbf{x} ; y_{1}\right) \mathrm{d} y_{1}=f_{0}^{\varepsilon} \mathbf{g}_{-3 / 2}\left(\mathbf{x} ; y_{1}\right)+\mathbf{g}_{-1 / 2}\left(\mathbf{x} ; y_{1}\right) \mathrm{d} y_{1} \\
\lim _{\mathbf{x} \rightarrow \mathbf{0}} \int_{0}^{\varepsilon} \mathbf{g}_{-1}\left(\mathbf{x} ; y_{1}\right) \mathrm{d} y_{1}=\frac{G}{2 \pi(1-\nu)}\left(\begin{array}{cc}
3 n_{2} & 3 n_{1} \\
3 n_{1} & -n_{2}
\end{array}\right)
\end{gathered}
$$

with:

$$
\begin{aligned}
& f_{0}^{\varepsilon} \mathbf{g}_{-3 / 2}\left(\mathbf{x} ; y_{1}\right)+\mathbf{g}_{-1 / 2}\left(\mathbf{x} ; y_{1}\right) \mathrm{d} y_{1}=
\end{aligned}
$$

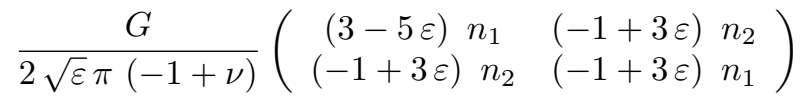

Proof: By direct integration.

In view of lemma 2.6, the approximation of integral (18) on $I_{\varepsilon}^{+}$is no longer $O(1)$ as for almost everywhere $C^{2}$ boundaries. In this case in fact a finite part of Hadamard concept comes into play, which turns out to be $O\left(\varepsilon^{-1 / 2}\right)$; its origin is totally different from the usual finite part integral in hypersingular integrals: it arises because the integral on the boundary is approximated by the integral on the tangent at the boundary. The term (24) is the counterpart of (20) for the curve $y_{2}=y_{1}^{3 / 2}$. To the best of my knowledge, this should be the first attempt to evaluate a free term on a $C^{1}$ only boundary. A general expression of such a free term, for at least a curve of type $y_{2}=y_{1}^{1+\beta}$ with $0<\beta<1$ is not available, so far; it will be the subject of further developments to the present note.

Propositions 2.4 and 2.5 apply as they stand also on $C^{1}$ boundaries. Denoting with $\mathcal{H}\left(y_{1}\right)$ the usual Heaviside function, proposition 2.6 may be extended as follows:

Proposition 2.8 If $y_{2}\left(y_{1}\right) \sim \alpha y_{1}^{3 / 2} \mathcal{H}\left(y_{1}\right)$ then

$$
\begin{aligned}
& \lim _{\mathbf{x} \rightarrow \mathbf{0}} \int_{\Gamma_{\varepsilon}} \mathbf{G}_{p p}(\mathbf{x}-\mathbf{y} ; \mathbf{n}(\mathbf{x}), \mathbf{l}(\mathbf{y})) \mathrm{d} \Gamma_{\mathbf{y}}= \\
& \quad \quad_{\Gamma_{\varepsilon}} \mathbf{G}_{p p}(-\mathbf{y} ; \mathbf{n}(\mathbf{0}), \mathbf{l}(\mathbf{y})) \mathrm{d} \Gamma_{\mathbf{y}}+\frac{G \alpha^{2}}{2 \pi(1-\nu)}\left(\begin{array}{cc}
3 n_{2} & 3 n_{1} \\
3 n_{1} & -n_{2}
\end{array}\right)
\end{aligned}
$$


Proof: From lemma 2.5 it comes out:

$$
\begin{aligned}
\lim _{\mathbf{x} \rightarrow \mathbf{0}} \int_{\Gamma_{\varepsilon}} \mathbf{G}_{p p}(\mathbf{x}-\mathbf{y} ; \mathbf{n}(\mathbf{x}), \mathbf{l}(\mathbf{y})) \mathrm{d} \Gamma_{\mathbf{y}}= \\
\quad \lim _{\mathbf{x} \rightarrow \mathbf{0}} \int_{I_{\varepsilon}^{+}} \mathbf{g}_{-1}\left(\mathbf{x} ; y_{1}\right) \mathrm{d} y_{1}+\lim _{\mathbf{x} \rightarrow \mathbf{0}} \int_{I_{\varepsilon}^{+}} \mathbf{g}_{-3 / 2}\left(\mathbf{x} ; y_{1}\right) \mathrm{d} y_{1}+ \\
+\lim _{\mathbf{x} \rightarrow \mathbf{0}} \int_{I_{\varepsilon}^{+}} \mathbf{g}_{-1 / 2}\left(\mathbf{x} ; y_{1}\right) \mathrm{d} y_{1}+\lim _{\mathbf{x} \rightarrow \mathbf{0}} \int_{I_{\varepsilon}^{+}} \mathbf{g}_{0}\left(\mathbf{x} ; y_{1}\right) \mathrm{d} y_{1}+ \\
+\lim _{\mathbf{x} \rightarrow \mathbf{0}}\left\{\int_{I_{\varepsilon}^{-}} \mathbf{G}_{p p}(\mathbf{x}-\mathbf{z} ; \mathbf{n}(\mathbf{x}), \mathbf{l}(\mathbf{z})) \mathrm{d} y_{1}+\int_{I_{\varepsilon}^{+}} \mathbf{G}_{p p}(\mathbf{x}-\mathbf{z} ; \mathbf{n}(\mathbf{x}), \mathbf{l}(\mathbf{z})) \mathrm{d} y_{1}\right\}
\end{aligned}
$$

by proposition 2.5 and lemma 2.6 (see also appendix 2):

$$
\begin{aligned}
& =\frac{G \alpha^{2}}{2 \pi(1-\nu)}\left(\begin{array}{cc}
3 n_{2} & 3 n_{1} \\
3 n_{1} & -n_{2}
\end{array}\right)+\lim _{\mathbf{x} \rightarrow \mathbf{0}} \int_{I_{\varepsilon}^{+}} \mathbf{g}_{0}\left(\mathbf{x} ; y_{1}\right) \mathrm{d} y_{1}+ \\
& \quad+f_{I_{\varepsilon}^{+}} \mathbf{g}_{-3 / 2}\left(\mathbf{x} ; y_{1}\right)+\mathbf{g}_{-1 / 2}\left(\mathbf{x} ; y_{1}\right) \mathrm{d} y_{1}+f_{I_{\varepsilon}} \mathbf{G}_{p p}(-\mathbf{y} ; \mathbf{n}(\mathbf{0}), \mathbf{l}(\mathbf{y})) \mathrm{d} \Gamma_{\mathbf{y}}
\end{aligned}
$$

and the thesis follows by noting that if $y_{2}\left(y_{1}\right) \sim \alpha y_{1}^{3 / 2} \mathcal{H}\left(y_{1}\right)$ :

$$
\begin{aligned}
& f_{\Gamma_{\varepsilon}} \mathbf{G}_{p p}(-\mathbf{y} ; \mathbf{n}(\mathbf{0}), \mathbf{l}(\mathbf{y})) \mathrm{d} \Gamma_{\mathbf{y}}=\lim _{\mathbf{x} \rightarrow \mathbf{0}} \int_{I_{\varepsilon}^{+}} \mathbf{g}_{0}\left(\mathbf{x} ; y_{1}\right) \mathrm{d} y_{1}+ \\
& \quad+f_{I_{\varepsilon}^{+}} \mathbf{g}_{-3 / 2}\left(\mathbf{x} ; y_{1}\right)+\mathbf{g}_{-1 / 2}\left(\mathbf{x} ; y_{1}\right) \mathrm{d} y_{1}+\oint_{I_{\varepsilon}} \mathbf{G}_{p p}(-\mathbf{y} ; \mathbf{n}(\mathbf{0}), \mathbf{l}(\mathbf{y})) \mathrm{d} \Gamma_{\mathbf{y}}
\end{aligned}
$$

Identity (16) holds as it stands also on every $C^{1}$ boundary.

\section{Free terms arising from the strongly singular kernel.}

This section aims to analyze the limit

$$
\lim _{\mathbf{x} \rightarrow \mathbf{x}_{0}}\left\{\int_{\Gamma_{\varepsilon}} \mathbf{G}_{p u}(\mathbf{r} ; \mathbf{n}(\mathbf{x})) \mathrm{d} \Gamma_{\mathbf{y}} \mathbf{p}\left(\mathbf{x}_{0}\right) \quad \mathbf{x} \in \Omega\right\}
$$

on a smooth, at least $C^{1}$, boundary $\Gamma_{\varepsilon}$.

Proposition 3.1 For solving integral (25) on smooth boundaries it is sufficient to solve integrals:

$$
\int_{x_{1}-\varepsilon}^{x_{1}+\varepsilon} \mathbf{G}_{p u}(\mathbf{r} ; \mathbf{n}(\mathbf{x})) \mathrm{d} r_{1} \quad \mathbf{x} \in \Omega
$$


Proof: $\quad$ The thesis follows immediately by assuming $\Gamma_{\varepsilon}$ as in proposition 2.1

Proposition 3.1 can be extended to less smooth boundary:

Proposition 3.2 If

$$
\sqrt{1+y_{2}^{\prime}\left(y_{1}\right)^{2}}=1+O\left(y_{1}\right) \quad \forall y_{1} \in[-\varepsilon, 0[\cup] 0, \varepsilon]
$$

then for solving integral (25) it is sufficient to solve integrals:

$$
\int_{x_{1}-\varepsilon}^{x_{1}} \mathbf{G}_{p u}(\mathbf{r} ; \mathbf{n}(\mathbf{x})) \mathrm{d} r_{1} \quad \mathbf{x} \in \Omega
$$

Proof: $\quad$ Denoting with $r=\|\mathbf{x}-\mathbf{y}\|$ and taking $\mathbf{x} \notin \Gamma_{\varepsilon}^{+}$, one writes in $\mathcal{L}^{+}$:

$$
\int_{\Gamma_{\varepsilon}^{+}} \frac{f(\mathbf{y})}{r} \mathrm{~d} \Gamma \stackrel{\text { def }}{=} \int_{s_{0}}^{l} \frac{f(\tilde{\mathbf{y}}(s))}{r} \mathrm{~d} s=\int_{0}^{\varepsilon} \frac{f\left(\mathbf{y}\left(y_{1}\right)\right)}{r} \mathrm{~d} y_{1}+O(\varepsilon)
$$

for any $f\left(\mathbf{y}\left(y_{1}\right)\right) \in C^{0}\left(I_{\varepsilon}^{+}\right)$in view of the adopted hypothesis.

Taking the limit to the boundary $\Omega \ni \mathbf{x} \rightarrow \mathbf{x}_{0} \in \Gamma_{\varepsilon}$, it comes out from [18]:

$$
\begin{aligned}
\lim _{\mathbf{x} \rightarrow \mathbf{x}_{0}} & \int_{x_{1}-\varepsilon}^{x_{1}+\varepsilon} \mathbf{G}_{p u}(\mathbf{r} ; \mathbf{n}(\mathbf{x})) \mathrm{d} r_{1}= \\
& +\lim _{\mathbf{x} \rightarrow \mathbf{x}_{0}}\left\{\log \|\mathbf{r}\| \mathbf{L}_{p u}+\arctan \left(\frac{r_{1}}{x_{2}}\right) \mathbf{A}_{p u}+\frac{1}{r^{2}} \mathbf{S}_{p u}\right\}_{r_{1}=x_{1}-\varepsilon}^{r_{1}=x_{1}+\varepsilon}
\end{aligned}
$$

where:

$$
\begin{aligned}
& \mathbf{L}_{p u}=-\frac{1}{4 \pi} \frac{1}{1-\nu}\left(\begin{array}{cc}
(3-2 \nu) n_{1} & (1-2 \nu) n_{2} \\
-(1-2 \nu) n_{2} & (1-2 \nu) n_{1}
\end{array}\right) \\
& \left.\lim _{\mathbf{x} \rightarrow \mathbf{x}_{0}} \frac{1}{r^{2}} \mathbf{S}_{p u}\right|_{r_{1}=x_{1}-\varepsilon} ^{r_{1}=x_{1}+\varepsilon}=\mathbf{0} \\
& \left.\lim _{\mathbf{x} \rightarrow \mathbf{x}_{0}} \arctan \left(\frac{r_{1}}{x_{2}}\right) \mathbf{A}_{p u}\right|_{r_{1}=x_{1}-\varepsilon} ^{r_{1}=x_{1}+\varepsilon}=\frac{1}{2} \frac{1}{1-\nu}\left(\begin{array}{cc}
(1-\nu) n_{2} & \nu n_{1} \\
(1-\nu) n_{1} & (1-\nu) n_{2}
\end{array}\right)(28)
\end{aligned}
$$

By introducing the concept of Cauchy principal value, see e.g. [6], the following identity can be proved in the local reference $\mathcal{L}$ [18]:

Proposition 3.3 For any $\varepsilon \in \mathbf{R}$ :

$$
\begin{aligned}
\lim _{\mathbf{x} \rightarrow \mathbf{x}_{0}} & \int_{x_{1}-\varepsilon}^{x_{1}+\varepsilon} \mathbf{G}_{p u}(\mathbf{r} ; \mathbf{n}(\mathbf{x})) \mathrm{d} r_{1}= \\
& \int_{x_{01}-\varepsilon}^{x_{01}+\varepsilon} \mathbf{G}_{p u}\left(\mathbf{x}_{0}-\mathbf{y} ; \mathbf{n}\left(\mathbf{x}_{0}\right)\right) \mathrm{d} r_{1}+\frac{1}{2}\left(\begin{array}{cc}
n_{2} & \frac{\nu}{1-\nu} n_{1} \\
n_{1} & n_{2}
\end{array}\right)
\end{aligned}
$$


Proof: $\quad$ Straightforward passages permit to obtain in the local reference $\mathcal{L}$ (see also [18]):

$$
\begin{aligned}
f_{x_{01}-\varepsilon}^{x_{01}+\varepsilon} \mathbf{G}_{p u}\left(\mathbf{x}_{0}-\mathbf{y} ; \mathbf{n}\left(\mathbf{x}_{0}\right)\right) \mathrm{d} r_{1}= \\
\quad-\left.\frac{1}{4 \pi} \frac{1}{1-\nu}\left(\begin{array}{cc}
(3-2 \nu) n_{1} & (1-2 \nu) n_{2} \\
-(1-2 \nu) n_{2} & (1-2 \nu) n_{1}
\end{array}\right) \log \|\mathbf{r}\|\right|_{r_{1}=-\varepsilon} ^{r_{1}=\varepsilon}=\mathbf{0}
\end{aligned}
$$

The thesis follows by comparison with (28).

Accordingly, the term due to the limit (28):

$$
\mathbf{D}_{p u}\left(\mathbf{x}_{0}, \mathbf{n}\left(\mathbf{x}_{0}\right)\right) \stackrel{\text { def }}{=} \frac{1}{2} \frac{1}{1-\nu}\left(\begin{array}{cc}
(1-\nu) n_{2} & \nu n_{1} \\
(1-\nu) n_{1} & (1-\nu) n_{2}
\end{array}\right)
$$

is the contribution of the strongly singular kernel to the free-term $\mathbf{D}(\mathbf{x})$ in equation (2). $\mathbf{D}_{p u}$ depends on the elastic properties of the body and on the selected direction $\mathbf{n}\left(\mathbf{x}_{0}\right)$ at the point $\mathbf{x}_{0} \in \Gamma_{\varepsilon}$ : when it is taken as the outward normal at $\mathbf{x}_{0}$, that is $\mathbf{n}\left(\mathbf{x}_{0}\right)=\mathbf{e}_{2}$, then $\mathbf{D}_{p u}=1 / 2 \mathbf{I}$ which is the well known amount of the free term coefficient for smooth boundaries.

\section{Properties of free terms coefficients}

As mentioned in sections 2.1 and 3 the free terms coefficients $\mathbf{D}_{p u}\left(\mathbf{x}_{0}, \mathbf{n}\left(\mathbf{x}_{0}\right)\right)$ and $\mathbf{D}_{p p}\left(\mathbf{x}_{0}, \mathbf{n}\left(\mathbf{x}_{0}\right)\right)$ depend on the elastic properties of the body and on the selected direction $\mathbf{n}\left(\mathbf{x}_{0}\right)$ at the point $\mathbf{x}_{0} \in \Gamma_{\varepsilon}$. An analysis of their behavior with respect to direction $\mathbf{n}\left(\mathbf{x}_{0}\right)$ is performed here in the local reference $\mathcal{L}$.

Proposition 4.1 On every smooth boundary, the free term coefficient $\mathbf{D}(\mathbf{x})$ in equation (2) is independent on $\mathbf{n}(\mathbf{x})$ and on the material properties of the body. Moreover, it holds

$$
\mathbf{D}(\mathbf{x})=\frac{1}{2} \mathbf{I}
$$

if $\mathbf{p}(\mathbf{x})$ in equation (2) refers to the direction $\mathbf{n}(\mathbf{x})$.

Proof: $\quad$ Free terms coefficients inherit from kernels $\mathbf{G}_{p u}$ and $\mathbf{G}_{p p}$ the linearity property with respect to $\mathbf{n}\left(\mathbf{x}_{0}\right)$, namely:

$$
\begin{aligned}
\mathbf{G}_{p u}(\mathbf{x}-\mathbf{y} ; \mathbf{n}(\mathbf{x})) & =n_{1}(\mathbf{x}) \mathbf{G}_{p u}\left(\mathbf{x}-\mathbf{y} ; \mathbf{e}_{1}\right)+n_{2}(\mathbf{x}) \mathbf{G}_{p u}\left(\mathbf{x}-\mathbf{y} ; \mathbf{e}_{2}\right) \\
\mathbf{G}_{p p}(\mathbf{x}-\mathbf{y} ; \mathbf{n}(\mathbf{x}) ; \mathbf{l}(\mathbf{y})) & =n_{1}(\mathbf{x}) \mathbf{G}_{p p}\left(\mathbf{x}-\mathbf{y} ; \mathbf{e}_{1} ; \mathbf{l}(\mathbf{y})\right)+n_{2}(\mathbf{x}) \mathbf{G}_{p p}\left(\mathbf{x}-\mathbf{y} ; \mathbf{e}_{2} ; \mathbf{l}(\mathbf{y})\right)
\end{aligned}
$$

Because at the outward normal $\mathbf{n}\left(\mathbf{x}_{0}\right)=\mathbf{l}\left(\mathbf{x}_{0}\right)=\mathbf{e}_{2}$ they hold:

$$
\mathbf{D}_{p u}\left(\mathbf{x}_{0}, \mathbf{e}_{2}\right) \mathbf{p}\left(\mathbf{x}_{0}, \mathbf{l}\left(\mathbf{x}_{0}\right)\right)=\frac{1}{2} \mathbf{p}\left(\mathbf{x}_{0}, \mathbf{e}_{2}\right) \quad \mathbf{D}_{p p}\left(\mathbf{x}_{0}, \mathbf{e}_{2}\right)=\mathbf{0}
$$


merely the case $\mathbf{n}\left(\mathbf{x}_{0}\right)=\mathbf{e}_{1}$ still needs to be investigated. For the strongly singular contribution one writes in such a case:

$$
\mathbf{D}_{p u}\left(\mathbf{x}_{0}, \mathbf{e}_{1}\right) \mathbf{p}\left(\mathbf{x}_{0}, \mathbf{l}\left(\mathbf{x}_{0}\right)\right)=\frac{n_{1}}{2} \frac{1}{1-\nu}\left(\begin{array}{cc}
0 & \nu \\
1-\nu & 0
\end{array}\right) \boldsymbol{\sigma}\left(\mathbf{x}_{0}\right) \mathbf{e}_{2}
$$

For the hypersingular contribution, after imposing the linear isotropic elastic constitutive law:

$$
\boldsymbol{\epsilon}=-\frac{\nu}{E} \operatorname{tr}(\boldsymbol{\sigma}) \mathbf{I}+\frac{1+\nu}{E} \boldsymbol{\sigma}
$$

and the following constraint:

$$
\sigma_{33}=\nu\left(\sigma_{11}+\sigma_{22}\right)
$$

which is due to the plain strain hypothesis, straightforward passages permit to state the following identity:

$$
\begin{aligned}
\mathbf{D}_{p p}\left(\mathbf{x}_{0}, \mathbf{e}_{1}\right) \mathbf{p}\left(\mathbf{x}_{0}, \mathbf{n}\left(\mathbf{x}_{0}\right)\right) & =-\frac{G}{1-\nu}\left(\begin{array}{ll}
1 & 0 \\
0 & 0
\end{array}\right) \boldsymbol{\epsilon}\left(\mathbf{x}_{0}\right) \mathbf{e}_{1} \\
& =-\frac{1}{2} \frac{1}{1-\nu}\left[(1-\nu) \sigma_{11}-\nu \sigma_{22}\right] \mathbf{e}_{1}
\end{aligned}
$$

One obtains therefore

$$
\mathbf{D}_{p u}\left(\mathbf{x}_{0}, \mathbf{e}_{1}\right) \mathbf{p}\left(\mathbf{x}_{0}, \mathbf{l}\left(\mathbf{x}_{0}\right)\right)-\mathbf{D}_{p p}\left(\mathbf{x}_{0}, \mathbf{e}_{1}\right) \mathbf{p}\left(\mathbf{x}_{0}, \mathbf{n}\left(\mathbf{x}_{0}\right)\right)=\frac{1}{2} \boldsymbol{\sigma} \mathbf{e}_{1}=\frac{1}{2} \mathbf{p}\left(\mathbf{x}_{0}, \mathbf{e}_{1}\right)
$$

By the linearity property of the kernels, one concludes therefore that:

$$
\mathbf{D}_{p u}\left(\mathbf{x}_{0}, \mathbf{n}\left(\mathbf{x}_{0}\right)\right) \mathbf{p}\left(\mathbf{x}_{0}, \mathbf{l}\left(\mathbf{x}_{0}\right)\right)-\mathbf{D}_{p p}\left(\mathbf{x}_{0}, \mathbf{n}\left(\mathbf{x}_{0}\right)\right) \mathbf{p}\left(\mathbf{x}_{0}, \mathbf{n}\left(\mathbf{x}_{0}\right)\right)=\frac{1}{2} \mathbf{p}\left(\mathbf{x}_{0}, \mathbf{n}\left(\mathbf{x}_{0}\right)\right)
$$

that is, the free term coefficient for the traction equation on smooth boundaries does not depend on the selected direction $\mathbf{n}\left(\mathbf{x}_{0}\right)$. By substituting (33) into equation (8), $\mathbf{D}\left(\mathbf{x}_{0}\right)$ amounts to:

$$
\mathbf{D}\left(\mathbf{x}_{0}\right)=\frac{1}{2} \mathbf{I}
$$

Remarks to proposition 4.1

- As a consequence of proposition 4.1, equation (2) holds as it stands for any direction $\mathbf{n}(\mathbf{x})$ on smooth boundaries, provided that $\mathbf{p}(\mathbf{x})$ refers to the direction $\mathbf{n}(\mathbf{x})$.

- In the proof of proposition 4.1, the constitutive equation (32) has been used. Accordingly, proposition 4.1 holds only for displacement and stress fields which fulfill equation (32).

Typically eq. (32) does not apply to the discrete approximations of the displacement field $\mathbf{u}(\mathbf{y})$ and of the traction field $\mathbf{p}(\mathbf{y})$, (say $\hat{\mathbf{u}}(\mathbf{y}), \hat{\mathbf{p}}(\mathbf{y})$ ) that pertain to the boundary element method. Accordingly, proposition 4.1 does not hold only for such discrete fields when $\mathbf{n}\left(\mathbf{x}_{0}\right) \neq \mathbf{l}\left(\mathbf{x}_{0}\right)$. 
Note, in fact, that at the outward normal $\mathbf{n}\left(\mathbf{x}_{0}\right)=\mathbf{l}\left(\mathbf{x}_{0}\right)=\mathbf{e}_{2}$, proposition 4.1 is proved in view of equation (31) and the constitutive law (32) is no longer required. Accordingly, the traction equation on the boundary (2) holds with $\mathbf{D}\left(\mathbf{x}_{0}\right)=\frac{1}{2} \mathbf{I}$ in the BEM solution along the boundary. On the contrary, the traction equation does not hold for the approximated stress tensor evaluation on the boundary as a post processing task (see section 5).

\section{Approximation of the free term}

Let $\hat{\mathbf{u}}(\mathbf{y}), \hat{\mathbf{p}}(\mathbf{y})$ be discrete approximations of the displacement field $\mathbf{u}(\mathbf{y})$ and of the traction field $\mathbf{p}(\mathbf{y})$, respectively:

$$
\hat{\mathbf{u}}(\mathbf{y})=\sum_{h=1}^{N_{u}} \boldsymbol{\psi}_{h}^{u}(\mathbf{y}) \hat{\mathbf{u}}_{h} \quad \hat{\mathbf{p}}(\mathbf{y})=\sum_{h=1}^{N_{p}} \boldsymbol{\psi}_{h}^{p}(\mathbf{y}) \hat{\mathbf{p}}_{h}
$$

The discretization of the unknown fields permits to transform the BIEs into sets of algebraic equations. Two main techniques have been successfully developed to this aim: the collocation [25] and the symmetric Galerkin [7] methods (SGBEM). The evaluation of the stress tensor is a "post-processing" task, after the determination of the unknown sets $\hat{\mathbf{u}}_{h}, \hat{\mathbf{p}}_{h}$.

The constitutive equation (32) does not apply to the approximation fields (34); therefore, the property of independence of $\mathbf{D}(\mathbf{x})$ on the direction $\mathbf{n}(\mathbf{x})$ and on the material properties of the domain, stated in section 4 for the problem solution, does not hold for the approximated evaluation of the stress tensor. To explain this fact by an example, consider the square domain of figure 4, with sides of length 2 . The lower horizontal side is constrained by $\overline{\mathbf{u}}(\mathbf{y})=\mathbf{0}$. The upper horizontal side is subjected to the vertical load $\overline{\mathbf{p}}(\mathbf{y})=\left(1+0.02 x_{2}\right)(\lambda+2 G) \mathbf{e}_{2}$, while the two vertical sides are loaded by $\overline{\mathbf{p}}(\mathbf{y})=\left(1+0.02 x_{2}\right) \lambda \mathbf{l}(\mathbf{y})$, denoting with $\lambda$ and $G$ the Lamè constants and by $\mathbf{l}(\mathbf{y})$ the outward normal. The analytical solution of the problem reads:

$$
\mathbf{u}(\mathbf{y})=x_{2}\left(1+0.01 x_{2}\right) \mathbf{e}_{2} \quad \boldsymbol{\sigma}(\mathbf{y})=\left(1+0.02 x_{2}\right)\left(\begin{array}{ccc}
\lambda & 0 & 0 \\
0 & \lambda+2 G & 0 \\
0 & 0 & \lambda
\end{array}\right)
$$

Having approximated the problem via the SGBEM through the discretization of figure 4 -a, the stress tensor has been evaluated at points $\mathbf{x}_{n}=\left\{1-0.1^{n}, 0.735\right\}$ : the stress component $\sigma_{22}\left(\mathbf{x}_{n}\right)$ is reported in table 1. Moreover, the stress tensor has been evaluated on the boundary at $\mathbf{x}_{\infty}=\{1,0.735\}$, by means of the traction equation (2) having set $\mathbf{D}\left(\mathbf{x}_{\infty}\right)=\frac{1}{2} \mathbf{I}$. Despite the sequence $\mathbf{x}_{n} \rightarrow \mathbf{x}_{\infty}$, table 1 and figure 4-b clearly show that $\boldsymbol{\sigma}\left(\mathbf{x}_{n}\right) \nrightarrow \boldsymbol{\sigma}\left(\mathbf{x}_{\infty}\right)$.

The approximated solution is obtained by using analytical integrations [18], therefore consistency errors cannot be invoked for the lack of convergence. Instead, it is due to the approximation of the free term coefficient $\mathbf{D}\left(\mathbf{x}_{\infty}\right)$, because equation (33) does not apply anymore. To figure out this last statement, define shape 


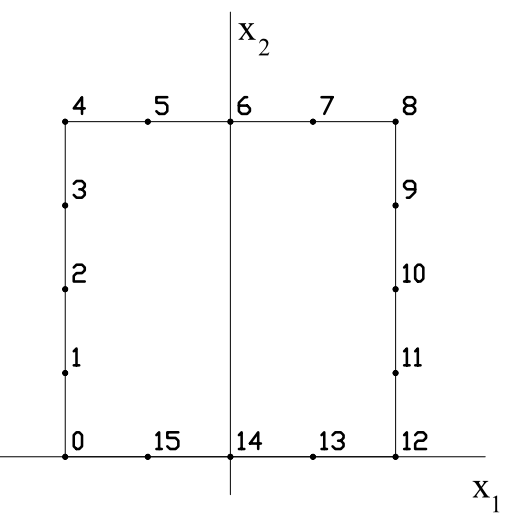

a) geometry and discretization by means of 16 equal linear boundary elements

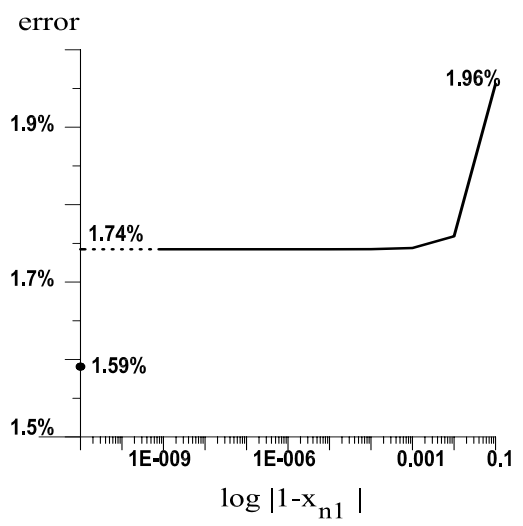

b) relative error at $\boldsymbol{\sigma}\left(\mathbf{x}_{\infty}\right)$ by evaluating it directly on the boundary by Eq. (2) and

by a limit process

Figure 4: A numerical benchmark for the stress tensor evaluation on the boundary.

\begin{tabular}{|c|c|c|c|}
\hline$x_{1}^{(n)}$ & $\sigma_{22}\left(\mathbf{x}_{n}\right)$ & $x_{1}^{(n)}$ & $\sigma_{22}\left(\mathbf{x}_{n}\right)$ \\
\hline 0.9 & 2782657.546 & 0.99 & 2782103.464 \\
\hline 0.999 & 2782060.611 & 0.9999 & 2782056.503 \\
\hline 0.99999 & 2782056.094 & 0.999999 & 2782056.053 \\
\hline 0.9999999 & 2782056.04922644 & 0.99999999 & 2782056.04881771 \\
\hline 0.999999999 & 2782056.04877683 & $\mathbf{x}_{\infty}$ & 2781629.623 \\
\hline
\end{tabular}

Table 1: Convergence of the stress tensor $\boldsymbol{\sigma}\left(\mathbf{x}_{n}\right)$.

functions $\psi_{h}^{u}(\mathbf{x})$ and $\psi_{h}^{p}(\mathbf{y})$ in equation (34) as follows. Let $\Gamma_{h}$ be a decomposition of the polygonal boundary $\Gamma$ of figure 4 with nodes $\left\{\mathbf{P}_{h}, h=1,2, \ldots, N_{h}\right\}$. Let $T_{j}$ be the generic segment of $\Gamma_{h}$, and let $l_{j}$ be half the length of $T_{j}$. Choose over $T_{j}$ a local basis $\left\{\varphi_{0}, \varphi_{1}, \ldots, \varphi_{N_{j}}\right\}$. Here $\varphi_{j}$ is a polynomial (usually lagrangian) of degree $N_{j}$ defined on a subset of $\left\{\mathbf{P}_{h}\right\}$ of $N_{j}+1$ nodes in $T_{j}$. Collect in set $\mathcal{T}_{h}=\left\{T_{j}\right\}_{j=1}^{N}$ the (two at most, i.e. $N \leq 2$ ) segments having the common vertex $\mathbf{P}_{h}$ (see figure 5); then $\psi_{h}$ is defined as:

$$
\psi_{h}(\mathbf{y}):= \begin{cases}\varphi_{n}(\mathbf{y}), & \mathbf{y} \in T_{j}, j=1, \ldots, N \\ 0, & \text { elsewhere }\end{cases}
$$

where the index $n$ selects the local basis function on $T_{j}$ such that $\varphi_{n}\left(\mathbf{P}_{h}\right)=1$. By construction, $\psi_{h}(\mathbf{y})$ is continuous over $\Gamma_{h}$, and its support coincides with $\mathcal{T}_{h}$.

Integrals in the HI (1), thus taking $\mathbf{x} \in \Omega$, take the form (see [18] for a larger 


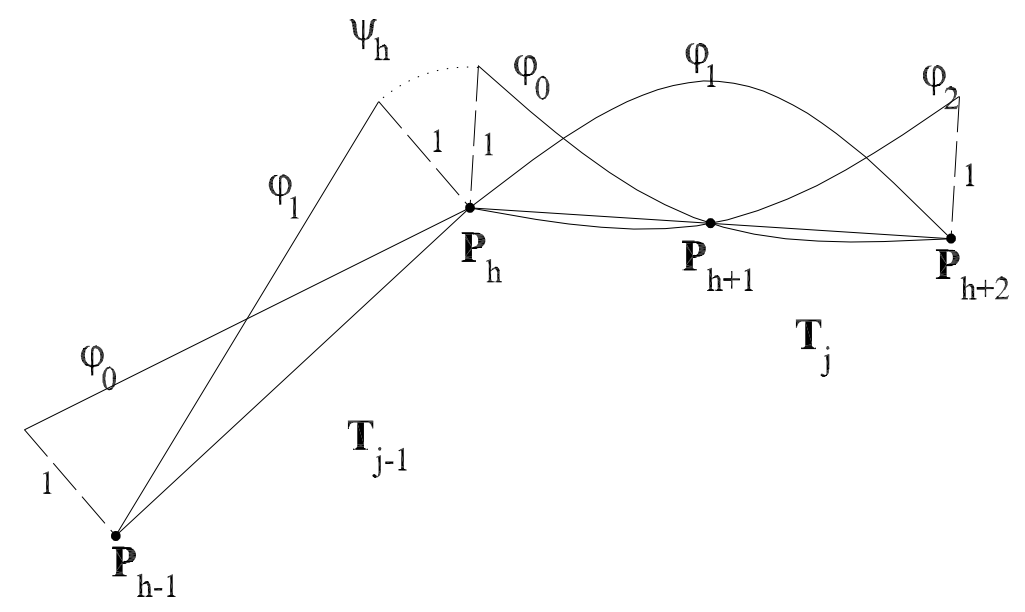

Figure 5: Local $\varphi_{n}(\mathbf{x})$ and global $\psi_{h}(\mathbf{x})$ shape function.

presentation) $s=u, p$ :

$$
\int_{\Gamma_{s}} \mathbf{G}_{p s}() \psi_{h}(\mathbf{y}) \mathrm{d} \Gamma_{\mathbf{y}}=\sum_{j=1}^{N} \mathbf{F}_{p s}^{j}(\mathbf{x}) \quad \mathbf{F}_{p s}^{j}(\mathbf{x}) \stackrel{\text { def }}{=} \int_{T_{j}} \mathbf{G}_{p s}() \varphi_{n}(\mathbf{y}) \mathrm{d} \Gamma_{\mathbf{y}}
$$

Denote by $\mathcal{L} \equiv\left\{y_{1}, y_{2}\right\}$ the local coordinate system defined in section 2 (see figure 2 ), with the origin in the midpoint of $T_{j}$. If $\mathbf{y} \in T_{j}$ then the outward normal $\mathbf{l}(\mathbf{y})=(0,1), y_{2}=0,-l_{j} \leq y_{1} \leq l_{j}$ and $\varphi_{n}(\mathbf{y})=\mathbf{y}_{1}^{T} \mathbf{a}_{n}$, having defined:

$$
\mathbf{y}_{1}^{T} \stackrel{\text { def }}{=}\left\{1, y_{1}, y_{1}^{2}, \ldots, y_{1}^{N_{j}}\right\} \quad \mathbf{a}_{n}^{T} \stackrel{\text { def }}{=}\left\{a_{n}^{(0)}, a_{n}^{(1)}, a_{n}^{(2)}, \ldots, a_{n}^{\left(N_{j}\right)}\right\}
$$

By means of the variable change $\mathbf{r}=\mathbf{x}-\mathbf{y}$ and the binomial expansion rule, it is straightforward to get $\mathbf{y}_{1}^{T}=\mathbf{r}_{1}^{T} \mathbf{X}$, where $\left(i, j=1,2, \ldots, N_{j}+1\right)$ :

$$
\mathbf{r}_{1}^{T} \stackrel{\text { def }}{=}\left\{1, r_{1}, r_{1}^{2}, \ldots, r_{1}^{N_{j}}\right\} \quad \mathbf{X}_{i j} \stackrel{\text { def }}{=}-(-1)^{i}\left(\begin{array}{c}
j-1 \\
i-1
\end{array}\right) x_{1}^{(j-i)}
$$

Equation (36) becomes therefore,

$$
\begin{aligned}
& \mathbf{F}_{p s}^{j}(\mathbf{x})=\mathbf{K}_{p s}^{j}(\mathbf{x})\left(\begin{array}{cc}
\mathbf{X} \mathbf{a}_{n} & 0 \\
0 & \mathbf{X} \mathbf{a}_{n}
\end{array}\right) \\
& \left.\mathbf{K}_{p s}^{j}(\mathbf{x}) \stackrel{\text { def }}{=} \int_{x_{1}-l_{j}}^{x_{1}+l_{j}} \mathbf{G}_{p s}()\left(\begin{array}{cc}
\mathbf{r}_{1}^{T} & 0 \\
0 & \mathbf{r}_{1}^{T}
\end{array}\right) \mathrm{d} r_{1}\right|_{r_{2}=x_{2}}
\end{aligned}
$$

with $\mathbf{x} \in \Omega$. Integrals $\mathbf{K}_{p s}^{j}$ have been analytically solved in [18]. They take the following expression in the local coordinate system $\mathcal{L}$ : 


$$
\begin{gathered}
\mathbf{K}_{p u}^{j}(\mathbf{x})=\frac{1}{4 \pi} \frac{1}{1-\nu} \\
{\left[\log \left(r^{2}\right) \mathbf{L}_{p u}+\arctan \left(\frac{r_{1}}{x_{2}}\right) \mathbf{A}_{p u}+\frac{1}{r^{2}} \mathbf{S}_{p u}+\mathbf{P}_{p u}\right]_{r_{1}=x_{1}-l_{j}}^{r_{1}=x_{1}+l_{j}}} \\
\mathbf{K}_{p p}^{j}(\mathbf{x})=\frac{G}{4 \pi} \frac{1}{1-\nu} \\
{\left[\log \left(r^{2}\right) \mathbf{L}_{p p}+\arctan \left(\frac{r_{1}}{x_{2}}\right) \mathbf{A}_{p p}+\frac{1}{r^{2}} \mathbf{S}_{p p}+\frac{1}{r^{4}} \mathbf{H}_{p p}+\mathbf{P}_{p p}\right]_{r_{1}=x_{1}-l_{j}}^{r_{1}=x_{1}+l_{j}}}
\end{gathered}
$$

where $r^{2}=r_{1}^{2}+x_{2}^{2}$ and $\mathbf{L}_{p u}, \mathbf{A}_{p u}, \mathbf{S}_{p u}, \mathbf{P}_{p u}, \mathbf{L}_{p p}, \mathbf{A}_{p p}, \mathbf{S}_{p p}, \mathbf{H}_{p p}$ and $\mathbf{P}_{p p}$ are suitable matrices, collected in [26] for $\varphi_{n}(\mathbf{y})$ of degree 5 . Moving $\mathbf{x}$ on the boundary, kernels $\mathbf{G}_{p u}(\mathbf{x}-\mathbf{y}, \mathbf{n}(\mathbf{x}))$ and $\mathbf{G}_{p p}(\mathbf{x}-\mathbf{y}, \mathbf{n}(\mathbf{x}), \mathbf{l}(\mathbf{y}))$ shows to be singular with respect to $\mathbf{y}$ depending on the position of $\mathbf{x}$ with respect to $T_{j}$.

With regard to the hypersingular kernel, by taking $\mathbf{x} \in T_{j}$ and by the definition of the finite part of Hadamard, it is straightforward to get:

$$
\begin{aligned}
\lim _{x_{2} \rightarrow 0^{-}} \mathbf{K}_{p p}^{j}(\mathbf{x}) & =f_{-l_{j}}^{l_{j}} \mathbf{G}_{p p}(\mathbf{x}-\mathbf{y} ; \mathbf{n}(\mathbf{x}) ; \mathbf{l}(\mathbf{y}))\left(\begin{array}{rrrr|r}
\mathbf{r}_{1}^{T} & 0 \\
0 & \mathbf{r}_{1}^{T}
\end{array}\right) \mathrm{d} y_{1}+ \\
& +\frac{G n_{1}}{(1-\nu)}\left[\begin{array}{llllll|l}
0 & 1 & 0 & \ldots & 0 & \mathbf{0} \\
\hline 0 & 0 & 0 & \ldots & 0 & \mathbf{0}
\end{array}\right]
\end{aligned}
$$

Analytical integrations suggest that the term

$$
\frac{G}{1-\nu}\left(\begin{array}{cc}
n_{1} & 0 \\
0 & 0
\end{array}\right)\left([0,1,0, \ldots, 0] \mathbf{X} \mathbf{a}_{n}\right) \hat{\mathbf{u}}_{h}=-\frac{G}{1-\nu}\left(\begin{array}{cc}
n_{1} & 0 \\
0 & 0
\end{array}\right) \frac{\mathrm{d} \varphi_{n}^{u}(\mathbf{x})}{\mathrm{d} x_{1}} \hat{\mathbf{u}}_{h}
$$

must be considered as an approximation of the hypersingular free term (17) on the boundary $\Gamma_{p}$ :

$$
\mathbf{D}_{p p}(\mathbf{x}, \mathbf{n}(\mathbf{x})) \mathbf{p}(\mathbf{x}, \mathbf{n}(\mathbf{x}))=-\frac{G}{1-\nu}\left(\begin{array}{cc}
n_{1} & 0 \\
0 & 0
\end{array}\right) \boldsymbol{\epsilon}\left(\mathbf{x}_{0}\right) \mathbf{e}_{1}
$$

It is evident that the isotropic linear constitutive law (32) can neither be applied to the approximated fields (34) nor to the free term approximation (39).

For the strongly singular kernel, a similar path of reasoning leads to the following identity:

$$
\begin{aligned}
\lim _{x_{2} \rightarrow 0^{-}} \mathbf{K}_{p u}^{j}(\mathbf{x}) & =\int_{-l_{j}}^{l_{j}} \mathbf{G}_{p u}(\mathbf{x}-\mathbf{y} ; \mathbf{n}(\mathbf{x}))\left(\begin{array}{cc}
\mathbf{r}_{1}^{T} & 0 \\
0 & \mathbf{r}_{1}^{T}
\end{array}\right) \mathrm{d} y_{1} \\
& +\frac{1}{2(1-\nu)}\left[\begin{array}{ccc|ccc}
n_{2}(1-\nu) & 0 & \ldots & n_{1} \nu & 0 & \ldots \\
\hline n_{1}(1-\nu) & 0 & \ldots & n_{2}(1-\nu) & 0 & \ldots
\end{array}\right]
\end{aligned}
$$

Analytical integrations suggest that the term

$$
\begin{aligned}
\frac{1}{2(1-\nu)}\left[\begin{array}{ccc|ccc}
n_{2}(1-\nu) & 0 & \ldots & n_{1} \nu & 0 & \ldots \\
\hline n_{1}(1-\nu) & 0 & \ldots & n_{2}(1-\nu) & 0 & \ldots
\end{array}\right]\left(\begin{array}{cc}
\mathbf{X} \mathbf{a}_{n} & 0 \\
0 & \mathbf{X} \mathbf{a}_{n}
\end{array}\right) \hat{\mathbf{p}}_{h}=(41) \\
\frac{1}{2(1-\nu)}\left(\begin{array}{cc}
n_{2}(1-\nu) & n_{1} \nu \\
n_{1}(1-\nu) & n_{2}(1-\nu)
\end{array}\right) \varphi_{n}^{p}(\mathbf{x}) \hat{\mathbf{p}}_{h}
\end{aligned}
$$


must be considered as an approximation of the free term (30) on the boundary $\Gamma_{u}$ :

$$
\mathbf{D}_{p u}(\mathbf{x}, \mathbf{n}(\mathbf{x})) \mathbf{p}(\mathbf{x}, \mathbf{l}(\mathbf{x}))=\frac{1}{2} \frac{1}{1-\nu}\left(\begin{array}{cc}
(1-\nu) n_{2} & \nu n_{1} \\
(1-\nu) n_{1} & (1-\nu) n_{2}
\end{array}\right) \mathbf{p}(\mathbf{x}, \mathbf{l}(\mathbf{x}))
$$

In the example studied above, for being $\mathbf{x}_{\infty} \in \Gamma_{p}$, the free term coefficient (42) was exactly evaluated. On the contrary, the hypersingular free term coefficient (40) was approximated by the term (39), thus giving rise to the emerged lack of convergence.

\section{Concluding remarks.}

In the present work the hypersingular formulation for boundary stress evaluation has been revised, exploiting recently developed analytical integration formulae [18]. Differently from the "vanishing exclusion zone" approach (see e. g. [15], [16]), in the present work: i) an asymptotic analysis of the singular integrals has been considered; ii) analytical integrations have been performed; iii) the limit to the boundary $\Omega \ni \mathbf{x} \rightarrow \mathbf{x}_{0} \in \Gamma$ has been taken. By this approach the contributions of the hypersingular kernel and of the strongly singular kernel to the free term coefficient $\mathbf{D}(\mathbf{x})$ have been separately obtained.

As a first conclusion of the present note, the hypersingular kernel contributes to the free term in the stress tensor evaluation on the boundary even when it is smooth. More generally, a hypersingular free term comes into play when the traction vector is evaluated with respect to a normal that differs from the boundary surface aside from the regularity of the boundary: such a phenomenon has been observed even on a straight boundary.

A deep investigation has been devoted to free terms coming out from the hypersingular kernel due to an "insufficient" smoothness of the boundary. It has been proved that free terms arise when the boundary is less smooth than $C^{2}$. Furthermore, a closed form free term has been evaluated for a boundary which is only $C^{1}$ smooth.

In such an analysis on the hypersingular integral operator, it has been proved that the concept of the finite part of Hadamard is consistent in the traction equation, in the sense that it comes out from a limit to the boundary process of the hypersingular integral. Such a consistency is questionable in the presence of corners, as will be seen in the companion paper [19], because the finite part formulation of the traction equation (2) in the presence of corners makes sense in view of a property of the global elastic integral operator but not for the hypersingular operator itself.

Furthermore, equation (2) has been proved to apply only to fields that comply with the constitutive law (32). For all fields that do not fulfill the constitutive law, equation (2) may not hold. For instance, equation (2) leads to the boundary element method by considering discrete approximation for displacement and trac- 
tion fields: for the discrete traction equation on the boundary, it has been proved that the free term coefficient on smooth boundaries does not hold $\frac{1}{2} \mathbf{I}$.

Such items are not only of theoretical interest: indeed the stress tensor on the boundary, which is the most interesting data in many problems of fracture propagation and bifurcation [10], [11], may largely differ from the expected value. As seen in an example, the error due to the free term approximation is of the same order of the error of the approximation scheme.

The present work is preliminary to the analysis of engineering problems, for which the stress tensor along the boundary is a fundamental item. In particular, problems of fracture initiation, propagation and bifurcation in soil-structure interactions [10], in composite [11] and biological materials and tissues are under investigation [27].

In a companion paper, which is the natural prosecution of the present one, an investigation of the integral formulation of the linear elastic problem is considered in the presence of corners, thus extending all results contained in the present work.

\section{Acknowledgements}

Part of the present work was performed in the first semester of 2008 at the Oak Ridge National Laboratory: I am grateful to Professor L.J.Gray for the opportunity of visiting and working with him. The support of the Italian Ministry of University and Research (MIUR) under grant ex 60\% - 2007: "Analisi non lineari

nella meccanica dei continui e della frattura mediante il metodo degli elementi al contorno" is gratefully acknowledged.

\section{References}

[1] F.J. Rizzo. An integral equation approach to boundary value problems of classical elastostatics. Quart. Appl. Math., 40:83-95, 1967.

[2] K.H. Hong and J.T. Chen. Derivations of integral equations of elasticity. ASCE J. Engrg. Mechanics, 114:1028-1044, 61988.

[3] O. Huber, A. Lang, and G. Kuhn. Evaluation of the stress tensor in 3D elastostatics by direct solving of hypersingular integrals. Computat. Mech., 12:39-50, 61993.

[4] M. Diligenti and G. Monegato. Finite-part integrals, their occurence and computation. Rend. Circ. Mat. Palermo, 33(II):39-61, 1993.

[5] J. Hadamard. Lectures on Cauchy's problem in linear partial differential equations. Yale Univ. Press, New Haven, Conn., USA, 1923.

[6] A.H. Zemanian. Distribution theory and transform analysis. Dover, 1987. 
[7] M. Bonnet, G. Maier, and C. Polizzotto. Symmetric Galerkin boundary element method. Applied Mechanical Review, 51:669-704, 1998.

[8] V. Mantič. On computing boundary limiting values of boundary integrals with strongly singular and hypersingular kernels in $3 \mathrm{~d}$ bem for elastostatics. Engng. Anal. Boundary Elem., 13:115-134, 1994.

[9] M. Guiggiani. Hypersingular formulation for boundary stress evaluation. Engng. Anal. Boundary Elem., 13:169-179, 1994.

[10] S.T. Slowik, J.M. Chandra Kishen, and V.E. Saouma. Mixed mode fracture of cementitious interfaces. Part 1: experimental results. Engineering Fracture Mechanics. Mech., 60:83-94, 1998.

[11] S.T. Smith and J.G. Teng. Interfacial stresses in plated beams. Engineering Structures, 23:857-871, 2001.

[12] C. Fiedler. On the calculation of boundary stresses with the Somigliana stress identity. Int. J. Numer. Methods Eng., 38:3275-3295, 1995.

[13] M. Guiggiani. Hypersingular boundary integral equations have an additional free term. Comp. Mech., 16:245-248, 1995.

[14] A. Young. A single domain bem for 3d elstostatic crack analysis using continuous elements. Int. J. Numer. Methods Eng., 39:1265-1293, 1996.

[15] M. Guiggiani. Formulation and numerical treatment of boundary integral equations with hypersingular kernels. In V. Sladek and J. Sladek, editors, Singular integrals in boundary element methods, Advances in Boundary Elements Series, pages 85-124. Computational Mechanics Publications, Southampton and Boston, 1998.

[16] V. Mantič and F. Paris. Existence and evaluation of the two free terms in the hypersingular boundary integral equation of potential theory. Engng. Anal. Boundary Elem., 16:253-260, 1995.

[17] P.A. Martin and F.J. Rizzo. Hypersingular integrals: how smooth must the density be? Int. J. Numer. Methods Eng., 39:687-704, 1996.

[18] A. Salvadori. Analytical integrations in 2D BEM elasticity. Int. J. Numer. Methods Eng., 53(7):1695-1719, 2002.

[19] A. Salvadori. Hypersingular formulation for boundary stress evaluation revisited. part 2: Corners. Technical Report 5, University of Brescia, 2006.

[20] L. Amerio. Analisi matematica, volume II. UTET, Milano, 1987. in italian.

[21] S. Miccoli. Spectral properties of Galerkin boundary element matrices. Comp. Mech., 25:578-589, 2000. 
[22] C. Schwab and W.L. Wendland. Kernel properties and representations of boundary integral operators. Mathematische Nachrichten, 156:187-218, 1992.

[23] G. Krishnasamy, F.J. Rizzo, and T.J. Rudolphi. Hypersingular boundary integral equations: Their occurrence, interpretation, regularization and computation. In P. K. Banerjee and S. Kobayashi, editors, Developments in Boundary Element Methods. Elsevier Applied Science Publishers, 1991.

[24] K. Toh and Mukherjee S. Hypersingular and finite part integrals in the boundary element method. Int. J. Solid Structures, 31:2299-2312, 1994.

[25] C.A. Brebbia, J.C.F. Telles, and L.C. Wrobel. Boundary Element Techniques. Springer-Verlag, Berlin, 1984.

[26] A. Salvadori. Quasi brittle fracture mechanics by cohesive crack models and symmetric Galerkin BEM. PhD thesis, Politecnico di Milano, 1999.

[27] A. Salvadori. A symmetric boundary integral formulation for cohesive interface problems. Computational Mechanics, 32(4-6):381-391, 2003.

\section{Appendix 1 - Function $\mathbf{g}_{-1}\left(\mathbf{x} ; y_{1}\right)$.}

$$
\begin{aligned}
& \mathrm{g}_{-1}\left(\mathbf{x} ; y_{1}\right)_{1,1} \stackrel{\text { def }}{=}-\left(y _ { 1 } \left(n _ { 1 } \left(-3 x_{1}{ }^{6}+3 x_{1}^{4} x_{2}{ }^{2}+7 x_{1}{ }^{2} x_{2}{ }^{4}+x_{2}{ }^{6}+\right.\right.\right. \\
& x_{1}\left(15 x_{1}^{4}+6 x_{1}^{2} x_{2}^{2}-17 x_{2}^{4}\right) y_{1}- \\
& 2\left(15 x_{1}^{4}+18 x_{1}{ }^{2} x_{2}{ }^{2}-5 x_{2}{ }^{4}\right) y_{1}{ }^{2}+6 x_{1}\left(5 x_{1}{ }^{2}+7 x_{2}{ }^{2}\right) y_{1}{ }^{3}- \\
& \left.15\left(x_{1}{ }^{2}+x_{2}{ }^{2}\right) y_{1}{ }^{4}+3 x_{1} y_{1}^{5}\right)+ \\
& n_{2} x_{2}\left(-6 x_{1}^{5}+21 x_{1}^{4} y_{1}-4 x_{1}^{3}\left(x_{2}^{2}+6 y_{1}^{2}\right)+\right. \\
& x_{1}^{2}\left(26 x_{2}^{2} y_{1}+6 y_{1}^{3}\right)- \\
& \left.\left.\left.3 y_{1}\left(x_{2}{ }^{4}-6 x_{2}{ }^{2} y_{1}{ }^{2}+y_{1}{ }^{4}\right)+2 x_{1}\left(x_{2}{ }^{4}-20 x_{2}{ }^{2} y_{1}{ }^{2}+3 y_{1}^{4}\right)\right)\right)\right) \\
& \mathrm{g}_{-1}\left(\mathbf{x} ; y_{1}\right)_{1,2} \stackrel{\text { def }}{=} y_{1}\left(n _ { 1 } x _ { 2 } \left(6 x_{1}{ }^{5}-21 x_{1}{ }^{4} y_{1}+4 x_{1}{ }^{3}\left(x_{2}{ }^{2}+6 y_{1}{ }^{2}\right)\right.\right. \\
& -2 x_{1}{ }^{2}\left(13 x_{2}{ }^{2} y_{1}+3 y_{1}^{3}\right)+ \\
& \left.3 y_{1}\left(x_{2}^{4}-6 x_{2}^{2} y_{1}^{2}+y_{1}^{4}\right)-2 x_{1}\left(x_{2}^{4}-20 x_{2}^{2} y_{1}^{2}+3 y_{1}^{4}\right)\right)- \\
& n_{2}\left(x_{1}{ }^{6}+x_{2}{ }^{6}-5 x_{1}{ }^{5} y_{1}-14 x_{2}{ }^{4} y_{1}{ }^{2}+9 x_{2}{ }^{2} y_{1}{ }^{4}-5 x_{1}{ }^{4}\left(x_{2}{ }^{2}-2 y_{1}{ }^{2}\right)+\right. \\
& 2 x_{1}^{3}\left(3 x_{2}{ }^{2} y_{1}-5 y_{1}^{3}\right)+x_{1}{ }^{2}\left(-5 x_{2}{ }^{4}+12 x_{2}{ }^{2} y_{1}{ }^{2}+5 y_{1}^{4}\right)+ \\
& \left.\left.x_{1}\left(19 x_{2}{ }^{4} y_{1}-22 x_{2}{ }^{2} y_{1}^{3}-y_{1}^{5}\right)\right)\right) \\
& \mathrm{g}_{-1}\left(\mathbf{x} ; y_{1}\right)_{2,1} \stackrel{\text { def }}{=} \mathrm{g}_{-1}\left(\mathbf{x} ; y_{1}\right)_{1,2} \\
& \mathrm{~g}_{-1}\left(\mathbf{x} ; y_{1}\right)_{2,2} \stackrel{\text { def }}{=} y_{1}\left(-\left(( x _ { 1 } { } ^ { 2 } + x _ { 2 } { } ^ { 2 } ) \left(2 n_{2} x_{1} x_{2}\left(x_{1}^{2}-3 x_{2}^{2}\right)+\right.\right.\right. \\
& \left.\left.n_{1}\left(x_{1}^{4}-6 x_{1}^{2} x_{2}^{2}+x_{2}^{4}\right)\right)\right)+
\end{aligned}
$$




$$
\begin{aligned}
& \left(n_{2} x_{2}\left(7 x_{1}^{4}+6 x_{1}^{2} x_{2}^{2}-9 x_{2}^{4}\right)+n_{1}\left(5 x_{1}^{5}-6 x_{1}^{3} x_{2}^{2}-19 x_{1} x_{2}^{4}\right)\right) y_{1}- \\
& 2\left(4 n_{2} x_{1} x_{2}\left(x_{1}^{2}+3 x_{2}^{2}\right)+n_{1}\left(5 x_{1}^{4}+6 x_{1}^{2} x_{2}^{2}-7 x_{2}^{4}\right)\right) y_{1}^{2}+ \\
& 2\left(5 n_{1} x_{1}{ }^{3}+n_{2} x_{1}{ }^{2} x_{2}+11 n_{1} x_{1} x_{2}{ }^{2}+7 n_{2} x_{2}{ }^{3}\right) y_{1}{ }^{3}- \\
& \left.\left(5 n_{1} x_{1}^{2}-2 n_{2} x_{1} x_{2}+9 n_{1} x_{2}^{2}\right) y_{1}^{4}+\left(n_{1} x_{1}-n_{2} x_{2}\right) y_{1}^{5}\right) \\
& \mathbf{g}_{-1}\left(\mathbf{x} ; y_{1}\right) \stackrel{\text { def }}{=} \frac{G}{\pi(-1+v)\left(x_{2}^{2}+\left(x_{1}-y_{1}\right)^{2}\right)^{4}} \mathbf{g}_{-1}\left(\mathbf{x} ; y_{1}\right)
\end{aligned}
$$

\section{Appendix 2 - Functions $\mathbf{g}_{i}\left(\mathbf{x} ; y_{1}\right)$.}

Function $\mathbf{g}_{-3 / 2}\left(\mathbf{x} ; y_{1}\right)$.

$$
\begin{aligned}
& \mathrm{g}_{-3 / 2}\left(\mathbf{x} ; y_{1}\right)_{1,1} \stackrel{\text { def }}{=}-2 n_{2} x_{2} y_{1}\left(5 x_{2}{ }^{4}-34 x_{2}{ }^{2} y_{1}{ }^{2}+9 y_{1}{ }^{4}\right)+ \\
& 3 n_{1}\left(x_{2}{ }^{6}+11 x_{2}{ }^{4} y_{1}{ }^{2}-21 x_{2}{ }^{2} y_{1}{ }^{4}+y_{1}{ }^{6}\right) \\
& \mathrm{g}_{-3 / 2}\left(\mathbf{x} ; y_{1}\right)_{1,2} \stackrel{\text { def }}{=}-2 n_{1} x_{2} y_{1}\left(5 x_{2}{ }^{4}-34 x_{2}{ }^{2} y_{1}{ }^{2}+9 y_{1}{ }^{4}\right)+ \\
& n_{2}\left(3 x_{2}{ }^{6}-51 x_{2}{ }^{4} y_{1}{ }^{2}+41 x_{2}{ }^{2} y_{1}{ }^{4}-y_{1}{ }^{6}\right) \\
& \mathrm{g}_{-3 / 2}\left(\mathbf{x} ; y_{1}\right)_{2,1} \stackrel{\text { def }}{=} \mathrm{g}_{-3 / 2}\left(\mathbf{x} ; y_{1}\right)_{1,2} \\
& \mathrm{~g}_{-3 / 2}\left(\mathbf{x} ; y_{1}\right)_{2,2} \stackrel{\text { def }}{=} 6 n_{2} x_{2} y_{1}\left(5 x_{2}{ }^{4}-10 x_{2}{ }^{2} y_{1}{ }^{2}+y_{1}^{4}\right)+ \\
& n_{1}\left(3 x_{2}{ }^{6}-51 x_{2}{ }^{4} y_{1}{ }^{2}+41 x_{2}{ }^{2} y_{1}{ }^{4}-y_{1}{ }^{6}\right) \\
& \mathbf{g}_{-3 / 2}\left(\mathbf{x} ; y_{1}\right) \stackrel{\text { def }}{=} \frac{\alpha G \sqrt{y_{1}}}{4 \pi(-1+\nu)\left(x_{2}^{2}+y_{1}^{2}\right)^{4}} \mathbf{g}_{-3 / 2}\left(\mathbf{x} ; y_{1}\right)
\end{aligned}
$$

Function $\mathbf{g}_{-1}\left(\mathbf{x} ; y_{1}\right)$.

$$
\begin{aligned}
& \mathrm{g}_{-1}\left(\mathbf{x} ; y_{1}\right)_{1,1} \stackrel{\text { def }}{=}-4 n_{2} x_{2} y_{1}\left(x_{2}{ }^{4}-10 x_{2}^{2} y_{1}^{2}+5 y_{1}^{4}\right)+ \\
& n_{1}\left(x_{2}{ }^{6}+15 x_{2}^{4} y_{1}^{2}-45 x_{2}^{2} y_{1}^{4}+5 y_{1}^{6}\right) \\
& \mathrm{g}_{-1}\left(\mathbf{x} ; y_{1}\right)_{1,2} \stackrel{\text { def }}{=}-4 n_{1} x_{2} y_{1}\left(x_{2}{ }^{4}-10 x_{2}{ }^{2} y_{1}{ }^{2}+5 y_{1}^{4}\right)+ \\
& n_{2}\left(x_{2}{ }^{6}-25 x_{2}{ }^{4} y_{1}^{2}+35 x_{2}^{2} y_{1}^{4}-3 y_{1}^{6}\right) \\
& \mathrm{g}_{-1}\left(\mathbf{x} ; y_{1}\right)_{2,1} \stackrel{\text { def }}{=} \mathrm{g}_{-1}\left(\mathbf{x} ; y_{1}\right)_{1,2} \\
& \mathrm{~g}_{-1}\left(\mathbf{x} ; y_{1}\right)_{2,2} \stackrel{\text { def }}{=} 4 n_{2} x_{2} y_{1}\left(3 x_{2}{ }^{4}-10 x_{2}{ }^{2} y_{1}{ }^{2}+3 y_{1}^{4}\right)+ \\
& n_{1}\left(x_{2}{ }^{6}-25 x_{2}{ }^{4} y_{1}{ }^{2}+35 x_{2}{ }^{2} y_{1}{ }^{4}-3 y_{1}{ }^{6}\right) \\
& \mathbf{g}_{-1}\left(\mathbf{x} ; y_{1}\right) \stackrel{\text { def }}{=} \frac{3 \alpha^{2} G x_{2} y_{1}^{2}}{2 \pi(-1+\nu)\left(x_{2}^{2}+y_{1}^{2}\right)^{5}} \mathbf{g}_{-1}\left(\mathbf{x} ; y_{1}\right)
\end{aligned}
$$


Function $\mathbf{g}_{-1 / 2}\left(\mathbf{x} ; y_{1}\right)$.

$$
\begin{aligned}
& \mathrm{g}_{-1 / 2}\left(\mathbf{x} ; y_{1}\right)_{1,1} \stackrel{\text { def }}{=} 4 n_{2} x_{2} y_{1}\left(-11 x_{2}{ }^{6}+159 x_{2}{ }^{4} y_{1}{ }^{2}-145 x_{2}{ }^{2} y_{1}{ }^{4}+5 y_{1}{ }^{6}\right)+ \\
& n_{1}\left(9 x_{2}{ }^{8}+184 x_{2}{ }^{6} y_{1}{ }^{2}-870 x_{2}{ }^{4} y_{1}{ }^{4}+240 x_{2}{ }^{2} y_{1}{ }^{6}+5 y_{1}{ }^{8}\right) \\
& \mathrm{g}_{-1 / 2}\left(\mathbf{x} ; y_{1}\right)_{1,2} \stackrel{\text { def }}{=} 4 n_{1} x_{2} y_{1}\left(-11 x_{2}{ }^{6}+159 x_{2}{ }^{4} y_{1}{ }^{2}-145 x_{2}{ }^{2} y_{1}{ }^{4}+5 y_{1}{ }^{6}\right)+ \\
& n_{2}\left(9 x_{2}{ }^{8}-336 x_{2}{ }^{6} y_{1}{ }^{2}+770 x_{2}{ }^{4} y_{1}{ }^{4}-168 x_{2}{ }^{2} y_{1}{ }^{6}-3 y_{1}{ }^{8}\right) \\
& \mathrm{g}_{-1 / 2}\left(\mathbf{x} ; y_{1}\right)_{2,1} \stackrel{\text { def }}{=} \mathrm{g}_{-1 / 2}\left(\mathbf{x} ; y_{1}\right)_{1,2} \\
& \mathrm{~g}_{-1 / 2}\left(\mathbf{x} ; y_{1}\right)_{2,2} \stackrel{\text { def }}{=} 4 n_{2} x_{2} y_{1}\left(33 x_{2}{ }^{6}-177 x_{2}{ }^{4} y_{1}{ }^{2}+107 x_{2}{ }^{2} y_{1}{ }^{4}-3 y_{1}{ }^{6}\right)+ \\
& n_{1}\left(9 x_{2}{ }^{8}-336 x_{2}{ }^{6} y_{1}{ }^{2}+770 x_{2}{ }^{4} y_{1}{ }^{4}-168 x_{2}{ }^{2} y_{1}{ }^{6}-3 y_{1}{ }^{8}\right) \\
& \mathbf{g}_{-1 / 2}\left(\mathbf{x} ; y_{1}\right) \stackrel{\text { def }}{=} \frac{\alpha^{3} G y_{1}{ }^{\frac{7}{2}}}{4 \pi(-1+\nu)\left(x_{2}^{2}+y_{1}^{2}\right)^{6}} \mathbf{g}_{-1 / 2}\left(\mathbf{x} ; y_{1}\right)
\end{aligned}
$$

